\title{
Resveratrol Alleviates Oxidative Stress Induced by Oxidized Soybean Oils and Improves Gut Function via Changing Gut Microbiota in Weaned Piglets
}

\section{Yanan Gao}

Northeast Agricultural University

Qingwei Meng

Northeast Agricultural University

\section{Xin Song}

Northeast Agricultural University

\section{Qianqian Zhao}

Northeast Agricultural University

Baoming Shi ( $\nabla$ shibaoming1974@163.com )

Institute of Animal Nutrition, Northeast Agricultural University https://orcid.org/0000-0001-8560-4564

\section{Research Article}

Keywords: Oxidative stress, Piglets, Resveratrol, Gut barrier, Short-chain fat acid, Gut microbiome

Posted Date: January 14th, 2022

DOI: https://doi.org/10.21203/rs.3.rs-1245019/v1

License: (c) (i) This work is licensed under a Creative Commons Attribution 4.0 International License. Read Full License 


\section{Abstract}

Background: The objective of this study was to investigate the effects of dietary resveratrol supplementation on growth performance, redox status, inflammatory state, and intestinal function of weaned piglets fed oxidized soybean oils.

Methods: A total of twenty-eight castrated weaned male piglets with a similar body weight of $10.19 \pm 1 \mathrm{~kg}$ were randomly assigned to 4 dietary treatments for 28 days feeding trial with 7 replications per treatment and 1 piglet per replicate. Treatments were arranged as a $2 \times 2$ factorial with oil type [fresh soybean oils (FSOs) vs. oxidized soybean oils (OSOs)] and dietary resveratrol (RES) (0 vs. $300 \mathrm{mg} / \mathrm{kg}$ ).

Result: Inclusion of OSOs decreased the villus/crypt ratio (VCR), while the villus height (VH) and VCR in the jejunum of weaned piglets was increased by dietary RES $(P<0.05)$. The activities of lipase, chymotrypsin, and lactase were decreased by OSOs, however dietary RES supplementation increased the activities of lipase, chymotrypsin, lactase, and a-amylase in the jejunum of weaned piglets $(P<0.05)$. Dietary RES increased the apparent digestibility of crude fat (EE). Dietary RES supplementation in the

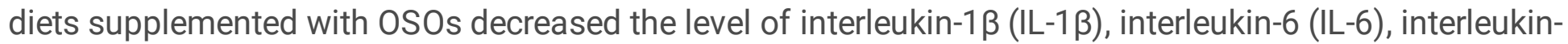
8 (IL-8), and tumor necrosis factor-a (TNF- $a$ ) in the plasma of weaned piglets, but failed to influence the IL-1 $\beta, I L-6, I L-8$, and TNF-a level when diets supplemented with FSOs. Dietary RES alleviated the decrease of total-superoxide dismutase activity in the plasma of weaned piglets fed OSOs $(P<0.05)$. Dietary supplemented with OSOs and RES decreased the level of $\mathrm{H}_{2} \mathrm{O}_{2}$ in the plasma of weaned piglets $(P<0.05)$. RES alleviates the intestinal barrier damage fed OSOs in weaned piglets by increasing the mRNA expression of ZO-1 and Occludin. It is noteworthy that inclusion of OSOs in diets increased the abundance of Actinobacteria, and decreased the abundance of Tenercutes $(P<0.05)$. RES increased the abundance of Firmicutes, and decreased the abundance of Bacteroidetes $(P<0.05)$. At the genus level, RES decreased the abundance of Prevotella-1, Prevotellaceae UCG003, and Clostridium_sensu_stricto_6 in the colon. OSOs decreased the level of acetic acid, and dietary RES increased the level of acetic acid and butyric acid in the colon of weaned piglets.

Conclusions: Dietary RES supplementation improved the villus-crypt structure, digestive enzyme activities and alleviated OSOs induced digestive absorption disorder. In addition, RES may alleviate OSOs immune status and energy metabolism of weaned piglets by affecting gut microbiota and its metabolite SCFAs. Notably, this positive effect of RES on OSOs may be related to decrease in the abundance of Prevotella_1 and Prevotellaceae_UCG-003.

\section{Background}

Dietary lipids give animals more time to digest and absorb other nutrients by slowing the rate of feed through the digestive tract ${ }^{1}$. In addition, as a vitamin vehicle, dietary lipids can effectively alleviate heat stress and provide a concentrated energy source for animals ${ }^{2}$. Soybean oil is a common source of dietary lipids added to animal diets. It is prone to lipid peroxidation, especially used for animal production in hot 
climates, because it contains a high concentration of unsaturated fatty acids ${ }^{3}$. It is well known that the presence of primary oxidation products will affect feed efficiency, which has a negative impact on animal performance ${ }^{4}$. Researchers have consistently demonstrated that the antioxidant status of swine ${ }^{5}$, broilers ${ }^{6}$, and rats $^{7}$, which feed oxidized oil is significantly lower than animals that fed normal oil. In addition, the presence of oxidized oil in swine feeds leads to increased oxidative susceptibility of pork products during storage ${ }^{8}$. Equally, weaning induces oxidative stress in piglets, which may be led to diarrhea, anorexia, intestinal barrier dysfunction, disturbance of free radicals and antioxidant system ${ }^{9,10}$, 11. It has been stated that weaned piglets fed oxidized soybean oil will exacerbates intestinal redox imbalance, inflammation, and intestinal barrier injury ${ }^{5}$. Similarly, a previous study demonstrated that inclusion of oxidized corn oil in diets decreased the average daily gain and average daily feed intake in finishing barrows ${ }^{10}$. Intestines are the primary site of pro-oxidation and anti-oxidation effects, exposure of animals to oxidized oil will increase the disruption of intestinal tissue structure, causing the imbalance of intestinal bacteria and increasing the likelihood of diarrhea and intestinal infections, and reducing the immunity and growth performance of animals ${ }^{7,12,13}$. Previous study demonstrated that piglets fed oxidized oil had a lower abundance of Prevotella_9 and higher abundances of Bacteroides and Campylobacter than control diet-fed piglets, and the concentrations of butyric acid and total SCFAs in colonic chyme were decreased ${ }^{14}$. Therefore, we hypothesized that intestinal dysfunction induced by oxidized oil consumption may be associated with the changes of intestinal microbiota. However, there are few types of research on the influence of feeding oxidized soybean oil on the intestinal microbiota in weaned piglets, and the biological mechanism to explain these phenomena remains unclear.

Accumulating evidence suggested that supplementation of additives with antioxidant function (such as short-chain fructans-oligosaccharides ${ }^{14}$, pterostilbene $^{15}$ ) in the feed allevates the oxidative stress and improves intestinal function of piglets. Phytogenic, a relatively new class of feed additives, comprises a wide variety of herbs, such as essential oils, polyphenols, etc ${ }^{15,16}$. In recent years, several studies have explored the relationship between polyphenolic compounds and gut bacteria as a new research perspective. Resveratrol (RES) is a C6-C2-C6 stilbene natural phenolic compound with three hydroxyl groups, several studies reported that RES has strong potential for promoting growth performance, antioxidative, anti-inflammatory by regulating the composition and content of the gut microbiota in animals undergoing stressful situations ${ }^{17,18,19}$. It has been stated that supplementation of RES in the diet of weaned piglets partially increased the jejunum villus height and tended to decrease the number of apoptotic cells in jejunum ${ }^{15}$. In addition, it has been reported that RES alleviates the intestinal inflammatory and improves intestinal immune function of piglets by improving the proliferation of beneficial bacteria Lactobacillus and Bifidobacterium, and inhibiting the proliferation of gram-negative bacteria ${ }^{20}$. Moreover, a previous study reported that dietary supplementation with RES alters the composition and metabolites of colonic microbiota in diquat-challenged piglets ${ }^{21}$. Hence, in the present study, we hypothesized that RES may ameliorate the oxidative stress induced by fed OSOs and improve intestinal health via modulating the gut microbiota of weaned piglets. 


\section{Materials And Methods}

\section{Animals, diets, and management}

The study was approved by the Animal Care and Use Committee of Northeast Agricultural University Institutional Animal Care and Use Committee (NEAU- [2011]-9). The animal experiment was conducted in Acheng Experimental Base of Northeast Agricultural University. A total of twenty-eight castrated weaned male piglets [Duroc $\times$ (Landrace $\times$ York-shire)] with a similar body weight of $10.19 \pm 1 \mathrm{~kg}$ was randomly assigned to 4 treatments with seven replications per treatment and 1 piglet per replicate for 28 days feeding trial. Treatments were arranged as a $2 \times 2$ factorial with oil type [FSOs vs. OSOs] and dietary RES (0 vs. $300 \mathrm{mg} / \mathrm{kg}$ ).

FSOs were purchased from Jiusan Grain and Oil Industry Group Co., Ltd. (Harbin, China). One part of fresh soybean oils was stored at $-20^{\circ} \mathrm{C}$ to prevent automat oxidation. The oxidization degree of soybean oils in different treatments was evaluated mainly by examining the peroxide value (POV). The OSOs were continuously treated with $\mathrm{FSOs}(\mathrm{POV}=11.7 \mathrm{mEqO} / \mathrm{kg})$ at $65^{\circ} \mathrm{C}$ and bubbled air at a rate of $4 \mathrm{~L} / \mathrm{min}$ until the POV reached approximately $384 \mathrm{mEqO}_{2} / \mathrm{kg}$, respectively. The fresh and oxidized soybean oils were stored at $-20^{\circ} \mathrm{C}$ for further experiments. POVs were examined as described by the American Oil Chemists Society ${ }^{22}$. Nutrient and energy densities of experimental diets, which were shown in Supplementary Table 1 , were set to meet, or exceed the nutritional requirements of swine from NRC (2012) ${ }^{2}$. All piglets were fed separately in a single stainless-steel metabolic cage containing a water dispenser and feeding tank for free drinking and feeding. During experiment, the pigpen temperature was kept between 20 and $23^{\circ} \mathrm{C}$. Meanwhile, the relative humidity was kept between 65 and $75 \%$. The light regime was a $12 \mathrm{~h}$ light/ $12 \mathrm{~h}$ dark cycle. The piglets had no access to probiotics and/or antibiotics throughout the entire experiment.

\section{Performance and diarrhea incidence}

After 28 days of feeding, piglets were fasted for $12 \mathrm{~h}$ before sacrifice. Body weights (BW) of piglets were individually evaluation on the mornings of day 1 , and day 29 of the feeding trial. Deed intake per pen was recorded daily throughout the trial to calculate average daily feed intake (ADFI), average daily gain (ADG) and average daily feed intake/average daily gain $(F / G)$. Clinical signs of diarrhea were visually assessed each morning by observers blinded to the treatments using a five-grade scoring system ${ }^{23}, 1=$ well-formed feces, 2 = slightly soft feces, 3 = soft and partially formed feces, 4 = loose and semiliquid feces, and 5 = watery feces. Then the average daily diarrhea index per replicating was calculated.

\section{Sample collection and processing}

On the 29th day of the trial, blood samples $(10 \mathrm{~mL})$ were collected using heparin tubes from piglets by venous puncture and then centrifuged at $3000 \mathrm{~g}$ for $15 \mathrm{~min}$ at $4^{\circ} \mathrm{C}$. The plasma was immediately collected and stored at $-20^{\circ} \mathrm{C}$ pending further analyses. After blood sampling, the piglets were sacrificed using electricity ( $250 \mathrm{~V}, 0.5 \mathrm{~A}$, for 5 to $6 \mathrm{~s}$ ), with subsequent jugular exsanguination. After opening the abdominal cavity, the gastrointestinal tract was removed to obtain portions of the jejunum, and the colon measuring approximately $20 \mathrm{~cm}$ was cut longitudinally and cleaned with ice-cold phosphate buffer 
solution (PBS). Mucosa samples were collected using scraping by sterile glass microscope slides. They were then snap-frozen in liquid nitrogen and stored at $-80^{\circ} \mathrm{C}$ for subsequent analysis. Two continuous segments were carefully cut from the middle of the whole jejunum and colon for histological assay and mucosa collection. Sections of approximately $1.5 \mathrm{~cm}$ in length were fixed in fresh, chilled $4 \%$ paraformaldehyde for morphometric evaluation and histochemical staining.

\section{Determination of apparent nutrient digestibility}

At the end of the experiment (day 24 to 27), feces from each group were collected for nutrient apparent digestibility determination, which was determined by the acid-insoluble ash method ${ }^{24}$. All the feces of piglets were collected twice a day at $8 \mathrm{~h}$ and $20 \mathrm{~h}$, put in plastic bags, weighed, and recorded. To prevent the loss of fecal ammonia, $10 \mathrm{mLH}_{2} \mathrm{SO}_{4}$ were added and the feces was stored in a $-20^{\circ} \mathrm{C}$ refrigerator. Before the samples were determined, the feces samples were thawed from the refrigerator and mixed evenly for four days from each pig. About $200 \mathrm{~g}$ of the mixed feces samples were taken out and dried in an oven at $60 \pm 5^{\circ} \mathrm{C}$ for $48-72 \mathrm{~h}$. Then the dried feces samples were crushed with a grinder and passed through a 40-mesh sieve for testing. Acid insoluble ash, dry matter (DM), crude protein (CP), crude fat (EE), gross energy (GE) in feces and feed were determined according to GB/T23742-2009 internal indicator method.

\section{Determination of digestive enzymes in the jejunum}

The sample preparation and the activities of jejunum digestive enzymes, including amylase (\#C016-1-1), lactase(\#A082-1-1), trypsin(\#A080-3-1), and lipase(\#A054-1-1), (\#A080-2-2), maltase(\#A082-3-1), sucrase(\#A082-2-1) of the jejunum were determined using commercial kits provided by Nanjing Jiancheng Bioengineering Institute (Jiangsu, China).

\section{Intestinal oxidative stress status and plasma cytokines analyses}

Based on the manufacturer's instructions, commercial kits were used to determine total superoxide dismutase (T-SOD \# A001-1-1), glutathione peroxidase (GSH-Px \#A005-1-2), hydrogen peroxide $\left(\mathrm{H}_{2} \mathrm{O}_{2}\right.$ \# A064-1-1), as well as total antioxidant capacity (T-AOC \# A015-1-2) and malondialdehyde (MDA \#A0031-2) were determined using commercial kits provided by Nanjing Jiancheng Bioengineering Institute (Jiangsu, China). Inflammatory cytokines in the plasma were determined with an ELISA kit (Beijing SinoUK Institute of Biological Technology.) according to the manufacturer's instructions.

\section{Determination of diamine oxidase (DAO) activity and D-lactate content in the plasma}

Plasma D-lactate and the diamine oxidase (DAO, \#A088-1-1) activity concentrations were determined using commercial ELISA kits provided by Nanjing Jiancheng Bioengineering Institute (Jiangsu, China). All procedures were performed with strict adherence to the manufacturer's guidelines.

\section{Mucosal morphometry and epithelial proliferation in jejunum and colon}


For intestinal morphological analysis, after being fixed in paraformaldehyde solution at room temperature for $24 \mathrm{~h}$, jejunal tissue specimens were dehydrated using an upgraded series of ethanol and xylene and then processed into paraffin blocks. A cross-section with a thickness of $5 \mu \mathrm{m}$ was cut from each specimen and stained with hematoxylin and eosin. Three of each group were randomly selected well-oriented and intact villi and adjacent crypts were randomly selected for the measurements of villus height $(\mathrm{VH})$ and crypt depth (CD) per slide by an assessor blinded to the treatments using optical microscopy (Nikon Eclipse 80i Nikon, Tokyo, Japan) and NIS-Elements 3.0 Imaging Software. Villus height needed to be evaluation in at least 10 villi from each pig and averaged. Data were analyzed using a digital microscope M8 and photographed at 40x magnification. The adjacent jejunum and colon were also fixed overnight in a 2.5.\% glutaraldehyde solution at $4{ }^{\circ} \mathrm{C}$, and then, these samples were treated for observation by electron microscopy. Epithelial proliferation was assessed using immunofluorescence in combination with a standardized quantification pipeline in jejunum and colon mucosa. To determine the relative amount of proliferative epithelial cells in the jejunum and colon mucosa samples, tissues were stained with nuclei stain PCNA and Hoechst ( $n=3$ randomly selected piglets from each treatment group).

\section{Immunofluorescence}

The jejunum and colon with $5 \%$ blank goat serum wax block sample complete coverage, slice to be placed in the wet box, at $37^{\circ} \mathrm{C}$ constant temperature and humidity incubator incubation for $30 \mathrm{~min}$. The blocking solution was removed, and the primary antibody working solution (ABclonal ZO-1, Occludin, Bioss, BSM-33070m, Ki67) prepared with antibody diluent was directly added to the samples. The samples were completely covered, and the sections were placed in a wet box and incubated at $4{ }^{\circ} \mathrm{C}$ overnight. Rewarming: the samples were placed at room temperature, rewarming for a $15 \mathrm{~min}$, antibody working solution was removed, and buffer solution in TBST was washed once for 5 minutes. Buffer solution TBS was washed for 3 times, 5 minutes each time, and the second antibody was incubated: the fluorescent second antibody working solution corresponding to the primary antibody species was dropped on the samples. The samples should be completely covered, protected from light, and incubated at $37^{\circ} \mathrm{C}$ for 1 hour. Remove the working solution of the secondary antibody and wash it with buffer TBST once for 5 minutes. Wash with buffer TBS 3 times, 5 minutes each time, dye the core: drop DAPI working solution on the sample, prepare with $0.01 \mathrm{M} \mathrm{pH7.2} \mathrm{TBS} \mathrm{buffer} \mathrm{solution,} \mathrm{DAPI} \mathrm{solution:} 0.01 \mathrm{M} \mathrm{pH7.2} \mathrm{TBS}$ buffer liquid volume ratio 1:500, avoid light, room temperature, incubated for 10 minutes; DAPI working solution was removed and washed with buffer TBST once for 5 minutes. Wash with buffer solution TBS for 3 times, 5 minutes each time, drop anti-fluorescence attenuation sealing tablets, then cover the cover glass sealing tablets, and observe under a fluorescence microscope and collect images.

\section{RNA extraction and RT-PCR}

The reaction system and the thermal cycling conditions used for RT-PCR were adjusted according to our previous study ${ }^{25}$. After retrieving the snap-frozen jejunum mucosal samples, we isolated total RNA using TRIzol Reagent (\#9109), as suggested in the manufacturer's manual (TaKaRa Biotechnology, Dalian, Liaoning, China). Extracted RNA was removed in $50 \mu \mathrm{L}$ of ultra-pure water. A NanoDrop ND-1000UV 
spectrophotometer (NanoDrop Technologies) was used to measure the purity and concentration of total RNA at 260 and $280 \mathrm{~nm}$. Electrophoresis on a 1.5\% agarose gel that had been stained Ultra GelRed ${ }^{\text {TM }}$ (\#GR501-01; Vazyme Biotech Co., Ltd.) was used to verify RNA integrity. One microgram of total RNA was then reverse-transcribed into complementary DNA using the Prime-Script ${ }^{\text {TM }}$ RT Reagent Kit (\#RR036A; TaKaRa Biotechnology). A real-time polymerase chain reaction (PCR) was performed on a QuantStudio 5 Real-Time PCR System (Applied Biosystems, Life Technologies, CA, USA) using the ChamQTM SYBR® qPCR Master Mix Kit (\#Q311-02; Vazyme Biotech Co., Ltd.), as recommended in the manufacturer's guidelines. The PCR process involved a 30 -s pre-run at $95^{\circ} \mathrm{C}, 40$ cycles of denaturation at $95^{\circ} \mathrm{C}$ for $5 \mathrm{~s}$, and a $60^{\circ} \mathrm{C}$ annealing step for $30 \mathrm{~s}$. For the melting curve conditions, one denaturation cycle was performed at $95^{\circ} \mathrm{C}$ for $10 \mathrm{~s}$; the temperature was then increased from 65 to $95^{\circ} \mathrm{C}$ at a rate $0.5^{\circ} \mathrm{C} / \mathrm{s}$. The primer sequences are shown in Supplementary Table 2 . The average of $\beta$-actin and GAPDH was used as

the internal control. The relative expression abundance of each target gene was calculated by the $2^{-\Delta \Delta} \mathrm{Ct}$ method, as elucidated previously ${ }^{26}$.

\section{Gut microbiota analysis}

Colonic digesta samples were collected from slaughtered piglets and immediately stored at $-80{ }^{\circ} \mathrm{C}$. Bacterial genomic DNA was extracted from each sample (Qiagen DNA Stool Mini Kit, Duesseldorf, Germany). DNA was quantified with a NanoDrop 2000 spectrophotometer (Thermo Scientific, Wilmington, NC, USA) and further assessed by running on $1 \%$ agarose gels. The V3-V4 hypervariable region of $16 \mathrm{~S}$ rRNA genes was amplified using specific primer pairs (forward 50-ACTCCTACGGGAGGCAGCA-30 and reverse 50-GGACTACHVGGGTWTCTAAT-30) with barcodes to construct the sequencing libraries (TruSeq ( $)$ DNA PCR-Free Sample Prep Kit, Illumina, San Diego, CA, USA). The qualified DNA libraries were loaded in a NovaSeq platform with $2 \times 250$ bp paired-end sequencing. The paired-end reads were obtained and merged using FLASH software (V1.2.7, http://ccb.jhu.edu/software /FLASH/). Operational taxonomic units (OTUs) with a 97\% identity were gathered with Unparsed (ver. 7.1, http://drive5.com/uparse /). Taxonomic annotation was performed using the Mothur algorithm ( $70 \%$ confidence) with the Silva Database (http://www.arb-silva.de/). The taxonomic composition of the bacterial community was then analyzed. RDA analyses were performed to identify the relationship among the identified differential genera of microbiota, growth performance, apparent digestibility, intestinal histomorphology, inflammatory cytokines, intestinal enzymatic activities, SCFAs, and treatments. Furthermore, according to the Pearson correlation analyses, several genera that were separately correlated with apparent digestibility, intestinal enzymatic activities and inflammatory cytokines, etc were also further identified.

\section{Quantification of SCFAs}

Short-chain fatty acids (SCFAs), including acetate, propionate, butyrate, valerate, is butyrate, and isovalerate, were quantified by an external standard method using gas chromatography (GC) as described by a previous study. Briefly, $1.5 \mathrm{~g}$ colonic chyme samples were added to screw-capped tubes with $6 \mathrm{~mL}$ of distilled water. After mixing overnight at $4{ }^{\circ} \mathrm{C}$ and centrifugation at $1500 \mathrm{r} / \mathrm{min}$ for $10 \mathrm{~min}$ at $4{ }^{\circ} \mathrm{C}, 2 \mathrm{~mL}$ of supernatant from each sample were transferred to another centrifuge tube, and $400 \mu \mathrm{L}$ of 
meta-phosphoric acid $(25 \% \mathrm{v} / \mathrm{v})$ were added to remove the protein. The samples were then centrifuged at $12,000 \mathrm{r} / \mathrm{min}$ for $10 \mathrm{~min}$ at $4{ }^{\circ} \mathrm{C}$. The resulting supernatants $(1 \mathrm{~mL}$ each) were transferred into gas chromatography sample bottles and analyzed using an Agilent 6890N GC (Palo Alto, CA, USA) coupled to a flame ionization detector with helium used as the carrier gas. An Agilent FFAP column $(30 \mathrm{~m} \times 0.53 \mathrm{~mm}$ i.d. $\times 1.00 \mu \mathrm{m}$ (film thickness)) was installed for analysis, with a constant flow rate of $4.0 \mathrm{~mL} / \mathrm{min}$. Spitless injection volume was $0.2 \mu \mathrm{L}$ of the sample. The injector and detector temperatures were $220^{\circ} \mathrm{C}$ and $240{ }^{\circ} \mathrm{C}$, respectively. The GC oven temperature was held at $90{ }^{\circ} \mathrm{C}$ for $1 \mathrm{~min}$ and then increased to 190 ${ }^{\circ} \mathrm{C}$ at a rate of $20^{\circ} \mathrm{C} / \mathrm{min}$ and held for $3 \mathrm{~min}$. Samples were run in triplicate, with a coefficient of variation less than $15 \%$ within triplicate samples used for quality control.

\section{Statistical analysis}

An individual pig was treated as the experimental unit. The statistical model included the fixed main effects of OSOs and RES and their interaction effects. Diarrhea score of piglets was analyzed using univariate ANOVA, data were analyzed using two-factor ANOVA for all other indicators (Version 23.0, SPSS Inc, Chicago, Illinois, USA), and visualized using GraphPad Prism. Semi-quantification of fluorescence was performed by Image $\mathrm{J}$ software. The data were expressed as the means \pm standard error of measurement (SEM), and a value of $(P<0.05)$ was considered statistically significant. Statistical differences were separated by Duncan's multiple range test. The significance level was set at $(P<0.05)$. Whereas $(0.05<P<0.10)$ was considered as a tendency.

\section{Results}

\section{Dietary RES alleviates intestinal morphology abnormalities and nutrient absorption disorders caused by inclusion of OSOs in the diets of weaned piglets}

As revealed by Table 1, inclusion of OSOs in the diet tended to decrease the average daily feed intake (ADFI) of weaned piglets $(P=0.060)$. But we observed that inclusion of OSOs or RES in diets failed to influence the initial weight (IW), average daily gain (ADG), average daily feed intake/the average daily gain $(F / G)$, and final weight (FW). The diarrhea score of weaned piglets was evaluated as shown in Fig. 1A. We observed that inclusion of OSOs in diets increased the diarrhea index during days 13-24 ( $P<$ $0.05)$. 
Table 1

Effects of OSOs and RES on growth performance of weaned piglets ${ }^{a, b}$

\begin{tabular}{|c|c|c|c|c|c|c|c|c|}
\hline \multirow[t]{3}{*}{ Item } & \multicolumn{2}{|l|}{ Fresh oil } & \multicolumn{2}{|c|}{ Oxidized oil } & \multirow[t]{3}{*}{ SEM } & \multicolumn{3}{|c|}{$P$-value } \\
\hline & $0 \mathrm{mg} / \mathrm{kg}$ & $300 \mathrm{mg} / \mathrm{kg}$ & $0 \mathrm{mg} / \mathrm{kg}$ & $300 \mathrm{mg} / \mathrm{kg}$ & & Oil & Res & Oil*Res \\
\hline & RES & RES & RES & RES & & & & \\
\hline IW (kg) & 10.15 & 10.23 & 10.2 & 10.19 & 0.10 & & & \\
\hline ADG $(\mathrm{kg})$ & 0.61 & 0.59 & 0.56 & 0.58 & 0.01 & 0.341 & 0.981 & 0.450 \\
\hline ADFI (kg) & 1.21 & 1.23 & 1.08 & 1.08 & 0.04 & 0.060 & 0.851 & 0.909 \\
\hline$F / G$ & 1.98 & 2.11 & 1.93 & 1.87 & 0.06 & 0.248 & 0.791 & 0.463 \\
\hline FW (kg) & 26.37 & 26.74 & 25.89 & 26.53 & 0.45 & 0.595 & 0.717 & 0.889 \\
\hline
\end{tabular}

The integrity of the intestinal structure is the basic guarantee of intestinal digestion and absorption of nutrients. The villus height $(\mathrm{VH})$, the crypt depth (CD), and the villus height: the crypt depth (VCR) of the intestinal are important indexes to measure intestinal digestion and absorption function ${ }^{27}$. As shown in Fig. 3, inclusion of OSOs decreased the VCR in the jejunum of weaned piglets. However, the VCR in the jejunum of weaned piglets was increased by dietary RES $(P<0.05)$. We observed that dietary RES supplementation in the diets supplemented with OSOs increased the $\mathrm{VH}$ in the jejunum of weaned piglets $(P<0.044)$, but failed to influence the VH when diets supplemented with FSOs (interaction, $P=0.016$ ). We observed that inclusion of OSOs or RES in diets failed to influence the CD in the jejunum or colon.

Furthermore, we evaluation the apparent digestibility of weaned piglets (Table 2). We found that dietary RES supplementation increased the digestibility of EE of weaned piglets $(P<0.05)$. Inclusion of OSOs or RES in diets failed to affect the digestibility of $D M, C P, G E$, and CF of weaned piglets $(P \otimes 0.05)$. These findings indicated that dietary RES improved the intestinal structure and digestive absorption function of weaned piglets. 
Table 2

Effects of OSOs and RES on nutrient apparent digestibility of weaned piglets $a, b$

\begin{tabular}{|c|c|c|c|c|c|c|c|c|}
\hline \multirow[t]{3}{*}{ Items } & \multicolumn{2}{|l|}{ Fresh oil } & \multicolumn{2}{|c|}{ Oxidized oil } & \multirow[t]{3}{*}{ SEM } & \multicolumn{3}{|c|}{$P$-value } \\
\hline & $0 \mathrm{mg} / \mathrm{kg}$ & $300 \mathrm{mg} / \mathrm{kg}$ & $0 \mathrm{mg} / \mathrm{kg}$ & $300 \mathrm{mg} / \mathrm{kg}$ & & Oil & Res & Oil*Res \\
\hline & RES & RES & RES & RES & & & & \\
\hline DM & 91.15 & 91.21 & 92.94 & 91.38 & 0.32 & 0.107 & 0.272 & 0.233 \\
\hline $\mathrm{CP}$ & 67.63 & 66.92 & 68.95 & 70.44 & 0.92 & 0.213 & 0.838 & 0.566 \\
\hline EE & 72.59 & 77.95 & 70.48 & 78.03 & 1.24 & 0.657 & 0.009 & 0.630 \\
\hline GE & 77.37 & 77.90 & 77.41 & 77.06 & 0.53 & 0.727 & 0.939 & 0.702 \\
\hline CF & 31.51 & 35.25 & 32.66 & 30.98 & 1.51 & 0.625 & 0.746 & 0.397 \\
\hline
\end{tabular}

Then, changes in small intestinal digestive enzymatic activities were further analyzed (as shown in Fig. 1B). We observed that the activities of lipase, chymotrypsin, and lactase activities were decreased by inclusion of OSOs, however, dietary RES supplementation increased the activities of lipase, chymotrypsin, lactase, and a-amylase in the jejunum of weaned piglets $(P<0.05)$. We observed that dietary RES supplementation in the diets supplemented with OSOs increased the activity of trypsin in the jejunum of weaned piglets $(P=0.015)$, but failed to influence the activity of trypsin when diets supplemented with FSOs (interaction, $P=0.031$ ). These results showed that dietary RES supplementation promotes the production of intestinal digestive enzymes and alleviates the digestive disorders induced by OSOs in weaned piglets. Considering the increased digestibility of fat, the genes related to fat transport in the intestine was evaluated. We found that the mRNA expression of jejunum absorption including FABP1 and the mRNA expression of jejunum absorption including FABP1, PPARY, CD36 were increased by dietary RES (Fig. 2) $(P<0.05)$.

\section{Dietary RES supplementation alleviates the oxidative stress state, inflammatory state, and intestinal barrier damage fed OSOs in weaned piglets}

The results of antioxidant status in the plasma of weaned piglets are shown in Table 3. Inclusion of OSOs increased the activity of total superoxide dismutase (T-SOD) in jejunum, while the T-SOD activity in the jejunum of weaned piglets was increased by RES supplementation ( $P>0.05$ ). Dietary RES supplementation in the diets supplemented with OSOs tended to decrease the level of $\mathrm{H}_{2} \mathrm{O}_{2}$ in the plasma and increase the contents of $\mathrm{H}_{2} \mathrm{O}_{2}$ in weaned piglets when diets supplemented with FSOs (interaction, $P=$ 0.015). The glutathione peroxidase (GSH-Px) activity, malondialdehyde (MDA) level, and total antioxidant capacity (T-AOC) were not influenced by inclusion of OSOs or RES ( $P>0.05)$. Furthermore, the genes 
related to antioxidant in the intestine were measure as shown in Fig. 2. We found that the mRNA expression of SOD2 in the jejunum and the mRNA expression of SOD2 and GPX1 in the colon were decreased by inclusion of OSOs, while dietary RES supplementation increased the mRNA expression of SOD2 in the jejunum and the mRNA expression of GPX1 in the colon of weaned piglets $(P<0.05)$, indicating that the oxidative stress state induced by OSOs was effectively alleviated by dietary RES.

Table 3

Effects of OSOs and RES on antioxidant capacity in the plasma of weaned piglets $a, b$

\begin{tabular}{|c|c|c|c|c|c|c|c|c|}
\hline \multirow[t]{3}{*}{ Items } & \multicolumn{2}{|c|}{ Fresh oil } & \multicolumn{2}{|c|}{ Oxidized oil } & \multirow[t]{3}{*}{ SEM } & \multicolumn{3}{|c|}{$P$-value } \\
\hline & $\begin{array}{l}0 \\
\mathrm{mg} / \mathrm{kg}\end{array}$ & $\begin{array}{l}300 \\
\mathrm{mg} / \mathrm{kg}\end{array}$ & $\begin{array}{l}0 \\
\mathrm{mg} / \mathrm{kg}\end{array}$ & $\begin{array}{l}300 \\
\mathrm{mg} / \mathrm{kg}\end{array}$ & & Oil & RES & Oil*RES \\
\hline & RES & RES & RES & RES & & & & \\
\hline $\mathrm{H}_{2} \mathrm{O}_{2}(\mathrm{mmol} / \mathrm{L})$ & $64.94^{b}$ & $74.93^{\mathrm{ab}}$ & $82.99^{a}$ & $70.11^{\mathrm{ab}}$ & 2.44 & 0.138 & 0.740 & 0.015 \\
\hline $\mathrm{GSH}-\mathrm{Px}(\mathrm{U} / \mathrm{mL})$ & 517.07 & 533.86 & 554.34 & 562.69 & 14.95 & 0.307 & 0.694 & 0.895 \\
\hline $\mathrm{T}-\mathrm{SOD}(\mathrm{U} / \mathrm{mL})$ & 46.69 & 68.29 & 71.61 & 88.19 & 5.12 & 0.019 & 0.040 & 0.772 \\
\hline $\begin{array}{l}\mathrm{MDA} \\
(\mathrm{nmol} / \mathrm{mL})\end{array}$ & 3.90 & 4.22 & 4.59 & 4.88 & 0.20 & 0.096 & 0.385 & 0.863 \\
\hline $\mathrm{T}-\mathrm{AOC}(\mathrm{U} / \mathrm{mL})$ & 0.36 & 0.34 & 0.38 & 0.37 & 0.01 & 0.143 & 0.978 & 0.783 \\
\hline
\end{tabular}

Furthermore, considering that the oxidative stress of the organism could induces the production of inflammatory, we further evaluated the inflammatory state of piglets ${ }^{10,11}$. As shown in Fig. 4A, dietary RES supplementation in the diets supplemented with OSOs decreased the level of IL-1 $\beta$, IL-6, IL-8, and TNF-a $(P<0.05)$ in the plasma of weaned piglets, but failed to influence the IL-1 $\beta$, IL-6, IL-8, and TNF-a level when diets supplemented with FSOs (interaction, $P<0.01$ ). As shown in Fig. 2 , the mRNA expression of pro-inflammatory cytokines, including IL-1 $\beta$ and TNF- $a$ in the jejunum of weaned piglets was increased by inclusion of OSOs in diets $(P<0.05)$. The mRNA expression of TNF- $\alpha$ and NF-KB were decreased by dietary RES supplementation $(P<0.05)$. Dietary RES supplementation increased the mRNA expression of $\mathrm{IL}-10$ in the jejunum of weaned piglets induced by OSOs $(P<0.05)$. The mRNA expression of TLR4 in the colon was decreased by dietary RES supplementation $(P<0.05)$. There was an interaction between OSOs and RES on IL-1 $\beta$ in the colon of weaned piglets. Dietary RES supplementation in the diets supplemented with OSO tended to decrease the mRNA expression of IL-1 $\beta$ in the colon of weaned piglets but failed to influence the mRNA expression of IL-1 $\beta$ when diets supplemented with FSOs (interaction, $P=0.029$ ). 
Intestinal inflammatory factors affect the function of tight junction protein, thus damaging the integrity of intestinal mucosa, while DAO activity and D-lactic content in plasma are important indicators of intestinal mucosal injury and permeability changes ${ }^{28,29}$. Therefore, we further studied the effects of OSO on the DAO activity and D-lactate content in the plasma of weaned piglets. The results of intestinal permeability biomarkers in plasma of weaned piglets are shown in Fig. 4B. Inclusion of OSOs in the diets increased the DAO activity in the plasma of weaned piglets, the DAO activity and D-lactate content in plasma of weaned piglets were decreased by dietary RES ( $P>0.05)$.

We further investigated the effects of dietary including OSOs on the intestinal barrier of weaned piglets. The expression of tight junction proteins in the jejunum and colon were detected (Fig. 2). The mRNA expression of ZO-1 in the jejunum and Occludin in the colon were decreased by dietary including OSOs of weaned piglets $(P<0.05)$. Dietary RES increased the mRNA expression of ZO- 1 and Occludin in jejunum and colon of weaned piglets $(P<0.05)$. But the mRNA expression of Claudin-5 and Claudin- 6 in jejunum and colon were not influenced by inclusion of OSOs or RES ( $P>0.05)$.

The results of immunofluorescence in jejunum and colon of weaned piglets are shown in Fig. 5. Inclusion of OSOs in the diets decreased the expression of Ki67 protein in the jejunum of piglets, the expression of ZO-1 in the jejunum of weaned piglets was increased by RES supplementation ( $P>0.05$ ). Inclusion of OSOs in the diet tended to decrease the expression of Occludin protein in the jejunum of weaned piglets $(P=0.055)$, the expression of Occludin in the jejunum of weaned piglets tended to increase by RES supplementation $(P=0.056)$. Dietary RES supplementation in the diets supplemented with OSOs failed to influence the expression of ZO-1 in the colon of weaned piglets but increased the expression of ZO-1 when diets supplemented with FSOs (interaction, $P=0.037$ ).

\section{Dietary RES affects the changes of gut microbiota and short-chain fatty acids (SCFAs) in the colon of inclusion of OSOs in the diets of weaned piglets}

As is shown in Fig. 6A. We observe that inclusion of OSOs in diets decreased the abundance of Tenercutes $(P<0.05)$. Dietary RES supplementation increased the abundance of Firmicutes $(P<0.05)$, and decreased the abundance of Bacteroidetes $(P<0.05)$. In addition, there was an interaction between inclusion OSOs or RES on the abundance of Actinobacteria in the colon of weaned piglets. Dietary RES in the diets supplemented with OSOs increased the abundance of Actinobacteria $(P=0.018)$ in the colon of weaned piglets but failed to influence the abundance of Actinobacteria when diets supplemented with FSOs (interaction, $P=0.005$ ). As shown in Fig. $6 \mathrm{~B}$, the gut microbiota composition at the genus level was further analyzed, the most dominant taxon was Prevotella_9 in all samples. Inclusion of OSOs in the diets increased the abundance of Parabacteroides in the colon of weaned piglets $(P<0.05)$. Dietary RES supplementation decreased the abundance of g_Clostridium_sensu_stricto_6 and PrevotellaceaeUCG003 in the colon of weaned piglets $(P<0.05)$. There was an interaction between OSOs and RES treatment for the abundance of Atopobiaceae_unclassified and Mollicutes_RF39_unclassified $(P<0.05)$. Dietary RES supplementation in the diets supplemented with OSOs tended to increase the abundance of Atopobiaceae_unclassified but tended to decrease the abundance of Atopobiaceae_unclassified in the 
colon of weaned piglets when diets supplemented with FSOs (interaction, $P=0.016$ ). Dietary RES supplementation in the diets supplemented with OSOs failed to influence the abundance of Mollicutes_RF39_unclassified ( $P=0.007$ ) but increased the abundance of Mollicutes_RF39_unclassified when diets supplemented with FSOs (interaction, $P=0.027)$. The alpha-diversity results of weaned piglets were not influenced by inclusion of OSOs or RES (Supplementary Table 3) ( $P>0.05$ ).

One of the microbial metabolites is SCFAs, which play an important role in the intestinal health as an energy source for the host ${ }^{30}$. Therefore, we further investigated the effects of dietary RES on SCFAs in colon contents of weaned piglets including OSOs in the diet. The level of SCFAs in the colon of weaned piglets are shown in Fig. 4C, inclusion of OSOs in the diet decreased the level of acetic acid and butyric acid in the colon of weaned piglets $(P<0.05)$. The level of acetic acid in the colon of weaned piglets was increased by dietary RES ( $P>0.05$ ). Dietary RES supplementation in the diets supplemented with OSOs increased the level of butyric acid in the colon of weaned piglets but failed to influence the level of butyric acid when diets supplemented with FSOs (interaction, $P=0.027$ ). The mRNA expression of G-proteincoupled receptor-41 (GPR41) was increased by RES in the colon of the weaned piglets $(P<0.05)$, but the mRNA expression of G- protein-coupled receptor-43 (GPR43) was not influenced by inclusion of OSOs or RES (Fig. 2B) ( $P>0.05)$.

\section{Changes in gut microbiota mediate the apparent digestibility, intestinal histomorphology, antioxygenic properties, inflammatory cytokines, and intestinal enzymatic activities in weaned piglets}

Diet is an important regulator of gut microbiome composition and diversity, which has beneficial effects on the host by regulating oxidative stress and metabolism ${ }^{31}$. RDA analyses were performed to identify the relationship among the identified differential genera of microbiota, weaned piglets' performance, and treatments (Fig. 7). We found that weaned piglets without RES supplementation were all separately clustered in the diarrhea score, while the weaned piglets with RES supplementation were not well clustered, which indicated that dietary RES supplementation is beneficial to reduce the diarrhea score of weaned piglets. The weaned piglets RES supplementation was aggregated near the EE, lipase, a-amylase, chymotrypsin, while the aggregation was not well in no RES supplementation weaned piglets. Indicating that dietary RES supplementation is beneficial to increase the lipase and EE. The weaned piglets RES supplementation was aggregated near the T-SOD, $\mathrm{H}_{2} \mathrm{O}_{2}$, indicating that dietary RES supplementation is beneficial to the improvement of the intestinal antioxidant capacity. The weaned piglets RES supplementation was aggregated near the IL-1 $\beta, \mathrm{IL}-6, \mathrm{IL}-8$, and TNF- $a$, indicating that dietary RES supplementation is beneficial to the recovery of the inflammatory state. The weaned piglets RES supplementation was aggregated near the acetic acid, butyric acid, and propionic acid. while the DAO and D-lactic aggregation were in weaned piglets NO RES. Furthermore, according to the Pearson correlation analyses, several key genera that were separately correlated with growth performance, nutrient apparent digestibility, intestinal histomorphology, antioxygenic properties, inflammatory cytokines, intestinal enzymatic activities, and SCFAs were also further identified and are listed in Table 4. Based on Pearson correlation analysis and RDA analysis, several different genera in the four treatment groups may affect the intestinal digestive enzyme activities, apparent digestibility, and pro-inflammatory factors. 
Specifically, of these genera, Prevotellaceae_UCG-003 and Prevotella_ 1 were negatively correlated with the increase of EE in weaned piglets. The abundances of Prevotellaceae_UCG_003 were negatively associated with the changes in a-amylase. The abundance of Prevotella_ 1 was positively associated with the changes in diarrhea rate13-16d. The abundance of Atopobiaceae_unclassified was negatively correlated with the increase of IL- 6 in the plasma of weaned piglets. 
Table 4

Pearson correlation analyses between key genera and growth performance, nutrient apparent digestibility, intestinal histomorphology, antioxygenic properties, inflammatory cytokines, intestinal enzymatic activities and SCAFs.

\begin{tabular}{|c|c|c|c|}
\hline Genus & Performance & Correlation coefficient & $P$-value \\
\hline Prevotellaceae_UCG-003 & a- amylase & -0.990 & 0.010 \\
\hline Prevotella_1 & EE & -0.981 & 0.019 \\
\hline Prevotellaceae_UCG-003 & $\mathrm{EE}$ & -0.966 & 0.034 \\
\hline Prevotella_1 & Diarrhea rate13-16d & 0.959 & 0.041 \\
\hline Atopobiaceae_unclassified & IL-6 & -0.954 & 0.046 \\
\hline Atopobiaceae_unclassified & IL-1 $\beta$ & -0.947 & 0.053 \\
\hline Prevotellaceae_UCG-003 & CD(Jejunum) & -0.941 & 0.059 \\
\hline Prevotella_1 & a- amylase & -0.914 & 0.086 \\
\hline Atopobiaceae_unclassified & TNF-a & -0.909 & 0.091 \\
\hline Mollicutes_RF39_unclassified & CD(Colon) & 0.902 & 0.098 \\
\hline Atopobiaceae_unclassified & IL-8 & -0.887 & 0.113 \\
\hline Clostridium_sensu_stricto_6 & Propionic acid & 0.869 & 0.131 \\
\hline Prevotella_1 & $\mathrm{VH}$ & -0.864 & 0.136 \\
\hline Mollicutes_RF39_unclassified & Lipase & 0.861 & 0.139 \\
\hline Parabacteroides & T-SOD & -0.844 & 0.156 \\
\hline Prevotellaceae_UCG-003 & Diarrhea rate13-16d & 0.843 & 0.157 \\
\hline Prevotella_1 & Chymotrypsin & -0.834 & 0.166 \\
\hline Prevotellaceae_UCG-003 & $\mathrm{VH}$ & -0.831 & 0.169 \\
\hline Prevotella_1 & CD(Jejunum) & -0.822 & 0.178 \\
\hline Atopobiaceae_unclassified & $\mathrm{VH}$ & 0.814 & 0.186 \\
\hline Prevotella_1 & DAO & 0.805 & 0.195 \\
\hline Mollicutes_RF39_unclassified & VCR & 0.790 & 0.210 \\
\hline Prevotella_1 & IL-8 & 0.771 & 0.229 \\
\hline Prevotella_1 & D-lactic acid & 0.760 & 0.240 \\
\hline Mollicutes_RF39_unclassified & Acetic acid & 0.757 & 0.243 \\
\hline Prevotella_1 & TNF-a & 0.749 & 0.251 \\
\hline
\end{tabular}




\begin{tabular}{|c|c|c|c|}
\hline Genus & Performance & Correlation coefficient & $P$-value \\
\hline Prevotellaceae_UCG-003 & IL-1 $\beta$ & 0.749 & 0.251 \\
\hline Prevotellaceae_UCG-003 & IL-8 & 0.746 & 0.254 \\
\hline Mollicutes_RF39_unclassified & D-lactic acid & -0.744 & 0.256 \\
\hline Atopobiaceae_unclassified & $\mathrm{H} 2 \mathrm{O} 2$ & -0.742 & 0.258 \\
\hline Prevotellaceae_UCG-003 & TNF-a & 0.737 & 0.263 \\
\hline Prevotella_1 & VCR & -0.725 & 0.275 \\
\hline Prevotella_1 & Diarrhea rate17-20d & 0.723 & 0.277 \\
\hline Prevotella_1 & IL-1 $\beta$ & 0.717 & 0.283 \\
\hline Parabacteroides & CD(Jejunum) & -0.714 & 0.286 \\
\hline Atopobiaceae_unclassified & CD(Jejunum) & 0.707 & 0.293 \\
\hline Prevotella_1 & Diarrhea rate21-24d & 0.706 & 0.294 \\
\hline Clostridium_sensu_stricto_6 & $\mathrm{H} 2 \mathrm{O} 2$ & -0.692 & 0.308 \\
\hline Clostridium_sensu_stricto_6 & T-SOD & -0.685 & 0.315 \\
\hline Mollicutes_RF39_unclassified & Chymotrypsin & 0.679 & 0.321 \\
\hline Prevotellaceae_UCG-003 & Chymotrypsin & -0.672 & 0.328 \\
\hline Mollicutes_RF39_unclassified & Propionic acid & -0.666 & 0.334 \\
\hline Parabacteroides & Acetic acid & 0.659 & 0.341 \\
\hline Prevotella_1 & Trypsin & -0.658 & 0.342 \\
\hline Mollicutes_RF39_unclassified & Butyric acid & 0.647 & 0.353 \\
\hline Parabacteroides & Butyric acid & 0.639 & 0.361 \\
\hline Prevotella_1 & Lipase & -0.629 & 0.371 \\
\hline Parabacteroides & CD(Colon) & 0.628 & 0.372 \\
\hline Prevotellaceae_UCG-003 & DAO & 0.622 & 0.378 \\
\hline Mollicutes_RF39_unclassified & Diarrhea rate17-20d & -0.618 & 0.382 \\
\hline Atopobiaceae_unclassified & a- amylase & 0.610 & 0.390 \\
\hline Prevotellaceae_UCG-003 & T-SOD & -0.607 & 0.393 \\
\hline Prevotellaceae_UCG-003 & D-lactic acid & 0.607 & 0.393 \\
\hline Prevotella_1 & Acetic acid & -0.589 & 0.411 \\
\hline
\end{tabular}




\begin{tabular}{|c|c|c|c|}
\hline Genus & Performance & Correlation coefficient & $P$-value \\
\hline Atopobiaceae_unclassified & Propionic acid & 0.584 & 0.416 \\
\hline Clostridium_sensu_stricto_6 & IL-6 & -0.573 & 0.427 \\
\hline Parabacteroides & a- amylase & -0.572 & 0.428 \\
\hline Prevotella_1 & CD(Colon) & -0.568 & 0.432 \\
\hline Prevotella_1 & IL-6 & 0.556 & 0.444 \\
\hline Parabacteroides & Lipase & 0.549 & 0.451 \\
\hline Prevotellaceae_UCG-003 & IL-6 & 0.547 & 0.453 \\
\hline Prevotella_1 & Butyric acid & -0.530 & 0.470 \\
\hline Mollicutes_RF39_unclassified & Diarrhea rate21-24d & -0.522 & 0.478 \\
\hline Prevotellaceae_UCG-003 & VCR & -0.521 & 0.479 \\
\hline Parabacteroides & VCR & 0.520 & 0.480 \\
\hline Atopobiaceae_unclassified & Trypsin & 0.514 & 0.486 \\
\hline Prevotella_1 & $\mathrm{H} 2 \mathrm{O} 2$ & 0.503 & 0.497 \\
\hline Prevotellaceae_UCG-003 & Diarrhea rate17-20d & 0.501 & 0.499 \\
\hline Mollicutes_RF39_unclassified & T-SOD & -0.494 & 0.506 \\
\hline Prevotellaceae_UCG-003 & Diarrhea rate21-24d & 0.491 & 0.509 \\
\hline Parabacteroides & Diarrhea rate17-20d & -0.491 & 0.509 \\
\hline Parabacteroides & IL-1 $\beta$ & 0.487 & 0.513 \\
\hline Clostridium_sensu_stricto_6 & D-lactic acid & 0.484 & 0.516 \\
\hline Prevotellaceae_UCG-003 & Trypsin & -0.470 & 0.530 \\
\hline Clostridium_sensu_stricto_6 & Trypsin & 0.461 & 0.539 \\
\hline Clostridium_sensu_stricto_6 & $\mathrm{EE}$ & -0.455 & 0.545 \\
\hline Parabacteroides & Diarrhea rate21-24d & -0.444 & 0.556 \\
\hline Prevotellaceae_UCG-003 & Lipase & -0.440 & 0.560 \\
\hline Prevotella_1 & Propionic acid & 0.427 & 0.573 \\
\hline Mollicutes_RF39_unclassified & DAO & -0.426 & 0.574 \\
\hline Clostridium_sensu_stricto_6 & Lipase & -0.425 & 0.575 \\
\hline Mollicutes_RF39_unclassified & IL-1 $\beta$ & 0.422 & 0.578 \\
\hline
\end{tabular}




\begin{tabular}{|c|c|c|c|}
\hline Genus & Performance & Correlation coefficient & $P$-value \\
\hline Mollicutes_RF39_unclassified & CD(Jejunum) & -0.410 & 0.590 \\
\hline Atopobiaceae_unclassified & Diarrhea rate13-16d & -0.409 & 0.591 \\
\hline Atopobiaceae_unclassified & DAO & -0.407 & 0.593 \\
\hline Mollicutes_RF39_unclassified & IL-6 & 0.401 & 0.599 \\
\hline Prevotella_1 & T-SOD & -0.376 & 0.624 \\
\hline Parabacteroides & D-lactic acid & -0.375 & 0.625 \\
\hline Prevotellaceae_UCG-003 & $\mathrm{H} 2 \mathrm{O} 2$ & 0.375 & 0.625 \\
\hline Prevotellaceae_UCG-003 & Propionic acid & 0.372 & 0.628 \\
\hline Atopobiaceae_unclassified & CD(Colon) & -0.365 & 0.635 \\
\hline Clostridium_sensu_stricto_6 & $\mathrm{CD}$ (Colon) & -0.363 & 0.637 \\
\hline Clostridium_sensu_stricto_6 & TNF-a & -0.362 & 0.638 \\
\hline Prevotellaceae_UCG-003 & $\mathrm{CD}$ (Colon) & -0.360 & 0.640 \\
\hline Clostridium_sensu_stricto_6 & IL-8 & -0.350 & 0.650 \\
\hline Parabacteroides & TNF-a & 0.349 & 0.651 \\
\hline Parabacteroides & Chymotrypsin & 0.344 & 0.656 \\
\hline Clostridium_sensu_stricto_6 & Chymotrypsin & -0.342 & 0.658 \\
\hline Mollicutes_RF39_unclassified & Diarrhea rate13-16d & -0.341 & 0.659 \\
\hline Prevotellaceae_UCG-003 & Acetic acid & -0.337 & 0.663 \\
\hline Atopobiaceae_unclassified & $\mathrm{EE}$ & 0.337 & 0.663 \\
\hline Clostridium_sensu_stricto_6 & Butyric acid & 0.334 & 0.666 \\
\hline Parabacteroides & IL-6 & 0.331 & 0.669 \\
\hline Clostridium_sensu_stricto_6 & a- amylase & -0.330 & 0.670 \\
\hline Atopobiaceae_unclassified & Lipase & -0.328 & 0.672 \\
\hline Clostridium_sensu_stricto_6 & IL-1 $\beta$ & -0.321 & 0.679 \\
\hline Parabacteroides & Trypsin & 0.315 & 0.685 \\
\hline Parabacteroides & IL-8 & 0.310 & 0.690 \\
\hline Mollicutes_RF39_unclassified & Trypsin & 0.310 & 0.690 \\
\hline Atopobiaceae_unclassified & Diarrhea rate21-24d & -0.310 & 0.690 \\
\hline
\end{tabular}




\begin{tabular}{|c|c|c|c|}
\hline Genus & Performance & Correlation coefficient & $P$-value \\
\hline Parabacteroides & DAO & -0.299 & 0.701 \\
\hline Mollicutes_RF39_unclassified & TNF-a & 0.293 & 0.707 \\
\hline Parabacteroides & $\mathrm{VH}$ & -0.283 & 0.717 \\
\hline Prevotellaceae_UCG-003 & Butyric acid & -0.277 & 0.723 \\
\hline Clostridium_sensu_stricto_6 & Diarrhea rate21-24d & -0.267 & 0.733 \\
\hline Clostridium_sensu_stricto_6 & CD(Jejunum) & -0.254 & 0.746 \\
\hline Mollicutes_RF39_unclassified & IL-8 & 0.244 & 0.756 \\
\hline Clostridium_sensu_stricto_6 & VCR & -0.242 & 0.758 \\
\hline Parabacteroides & Propionic acid & -0.239 & 0.761 \\
\hline Parabacteroides & $\mathrm{EE}$ & -0.235 & 0.765 \\
\hline Mollicutes_RF39_unclassified & a- amylase & -0.218 & 0.782 \\
\hline Atopobiaceae_unclassified & T-SOD & 0.215 & 0.785 \\
\hline Clostridium_sensu_stricto_6 & VH & 0.211 & 0.789 \\
\hline Clostridium_sensu_stricto_6 & DAO & -0.203 & 0.797 \\
\hline Atopobiaceae_unclassified & Diarrhea rate17-20d & -0.195 & 0.805 \\
\hline Atopobiaceae_unclassified & D-lactic acid & 0.193 & 0.807 \\
\hline Mollicutes_RF39_unclassified & $\mathrm{EE}$ & 0.180 & 0.820 \\
\hline Clostridium_sensu_stricto_6 & Diarrhea rate17-20d & -0.144 & 0.856 \\
\hline Mollicutes_RF39_unclassified & $\mathrm{VH}$ & -0.142 & 0.858 \\
\hline Atopobiaceae_unclassified & Butyric acid & 0.142 & 0.858 \\
\hline Clostridium_sensu_stricto_6 & Acetic acid & 0.131 & 0.869 \\
\hline Atopobiaceae_unclassified & VCR & -0.118 & 0.882 \\
\hline Parabacteroides & $\mathrm{H} 2 \mathrm{O} 2$ & -0.101 & 0.899 \\
\hline Clostridium_sensu_stricto_6 & Diarrhea rate13-16d & 0.099 & 0.901 \\
\hline Parabacteroides & Diarrhea rate13-16d & -0.063 & 0.937 \\
\hline Mollicutes_RF39_unclassified & $\mathrm{H}_{2} \mathrm{O}_{2}$ & 0.028 & 0.972 \\
\hline Atopobiaceae_unclassified & Chymotrypsin & -0.024 & 0.976 \\
\hline Atopobiaceae_unclassified & Acetic acid & 0.005 & 0.995 \\
\hline
\end{tabular}




\section{Discussion}

Intestinal morphology, an important indicator of intestinal health, is usually evaluated according to $\mathrm{VH}$, $\mathrm{CD}$, and $\mathrm{VCR}{ }^{32}, 33$. This study revealed that OSOs in the diet decreased the VCR in the colon and that dietary RES effectively alleviated gut morphology of weaning piglets, including VH and VCR in the jejunum of the weaned piglet. Previous studies have reported that RES restored damaged villus-crypt structure and improved gut absorption in pigs and broilers ${ }^{18,34}$. In this study, the lipase, chymotrypsin, and lactase activities were observed to decrease due to OSOs in the diets, and dietary RES increased the activities of lipase, chymotrypsin, and a-amylase in the jejunum of weaned piglets, indicating that supplementation of RES alleviated nutrient digestibility of weaned piglets fed diets including OSOs by restoring villus-crypt structure and enhancing digestive enzymes. In addition, as the gut is vulnerable to oxidative damage, lipid peroxidation products ingested by animals could often disrupt the redox balance and induce inflammation, resulting in a lack of antioxidant systems in the gut and other tissues ${ }^{35,36}$. According to this study, dietary RES further decreased the T-SOD activity in the plasma of weaned piglets induced by OSOs. SOD catalyzes the reduction of superoxide anion to hydrogen peroxide, which is further decomposed into water and oxygen by catalase, protecting cells from oxidative damage and scavenging free radicals in the body to maintain cell energy metabolism ${ }^{37}$. Dietary RES with OSOs reduced the $\mathrm{H}_{2} \mathrm{O}_{2}$ in the plasma of weaned piglets, a major ROS synthesized by a mutational reaction catalyzed by SOD and reduced to water by GSH-Px or CAT. In addition, as a central redox signaling molecule, $\mathrm{H}_{2} \mathrm{O}_{2}$ induced oxidative stress in the gut epithelial ${ }^{38,39}$. Therefore, the inhibition of $\mathrm{H}_{2} \mathrm{O}_{2}$ level and the increase of T-SOD activity could be the reasons why dietary RES alleviated the beneficial effects of oxidative stress in weaned piglets fed diets with OSOs. Besides, dietary RES increased the SOD2 mRNA expression in the jejunum. Previous studies have also observed that RES improved the activities of various antioxidant enzymes ${ }^{39,40}$. In this study, OSOs increased the activity of DAO, while RES decreased the DAO activity and D-lactate content in the plasma of weaned piglets. D-lactic and DAO, chemical markers of gut permeability, were released into the bloodstream when the intestinal barrier function was impaired ${ }^{28,}{ }^{29}$. In addition, OSOs in the diet of weaned piglets decreased the mRNA expression of Occludin in the colon and ZO-1 in the jejunum and colon. The protein expression of Occludin in the jejunum of weaned piglets tended to decrease due to the inclusion of OSOs in the diet, while RES increased the protein expression of ZO-1 in the jejunum of weaned piglets. The protein expression of ZO-1 was in accord with the mRNA expression of ZO-1.

Similarly, previous studies demonstrated that the pretreatment of cultured cells with RES prevented hydrogen peroxide-induced epithelial barrier damage in vitro ${ }^{41}$. Involved in the assembly and stability of tight junctions, Occludin and ZO-1 were proteins with the best barrier function ${ }^{42}$. Ki-67 was a specific and reliable marker of cell proliferation ${ }^{43}$, and OSOs decreased its protein expression in the jejunum of weaned piglets. Therefore, dietary RES with OSOs improved gut permeability, which might enhance ZO-1 and Ki-67 expression. These results further confirmed that RES improved the gut barrier damage of weaned piglets containing OSOs in the diets. 
The gut microbiome largely affects the health and survival of the host by regulating nutrient metabolism, immune system maturation, and gut barrier establishment ${ }^{31,44,45}$. Based on previous studies, body weight and gut microbial ecology were correlated. Obese subjects had an increased proportion of Firmicutes and a decreased proportion of Bacteroidetes ${ }^{46}$, indicating that RES could promote the growth of weaned piglets by raising the Firmicutes/Bacteroides ratio. However, OSOs and RES failed to affect the growth performance of weaned piglets, probably because only 7 repeated sample sizes in this study ( $\mathrm{n}=$ 7) were suitable for testing the effects of RES on gut barrier function and immune response in weaned piglets with diets including OSOs. Continuous testing should be conducted on more piglets to specify the growth performance. In our study, OSOs decreased the abundance of Tenericutes in the colon of piglets. Previous studies showed that oxidative stress reduced the abundance of Tenericutes in rats, which were associated with increased apparent digestibility of crude fiber in animals, and that healthy people had higher levels of Tenericutes than metabolically disturbed individuals $47,48,49$. Moreover, dietary RES supplemented with OSOs raised the abundance of Actinobacteria but failed to work when FSOs were added to the diets. Actinobacteria as part of the normal gut microbiota were rarely observed in the gut microbiota of different gastrointestinal disease ${ }^{50}$. From these results, dietary RES supplementation alleviated some gastrointestinal diseases caused by the oxidative stress-induced decrease of Tenericutes abundance in weaned piglets via increasing the abundance of Actinobacteria. Interestingly, OSOs and RES exposure failed to affect the alpha-diversity of the gut microbiome of piglets, suggesting similar ecological diversity of the colonic microbiota of piglets after different treatments, which was consistent with the previous research ${ }^{40}$.

In this study, dietary RES decreased colonic abundance of the Clostridium_sensu_stricto_6 of weaned piglets, identified as a common bacteria leading to diarrhea ${ }^{51,52}$. OSOs decreased the abundance of Parabacteroides in the intestines of piglets. The previous study demonstrated that Parabacteroides reduced the metabolic dysfunction of mice on a high-fat diet by converting bile acids and generating succinic acid $^{53}$. Furthermore, our study found that RES increased the apparent fat digestibility of EE in weaned piglets, which could be related to the increase of bile secretion ${ }^{54}$. Therefore, we hypothesized that RES increased the apparent digestibility of EE in weaned piglets due to changes in the abundance of Paracteroides. Prevotella, an active hemicellulose-decomposing bacterium, is necessary for degrading plant non-fiber polysaccharides and proteins ${ }^{55,56}$. Based on our work, dietary RES decreased the abundance of Prevotella-1 and Prevotellaceae UCGO03 in the colon of weaned piglets. However, increasing evidence suggested that Prevotella was also related to inflammatory conditions in the intestine ${ }^{57,58}$. Several studies reported that Prevotella could stimulate epithelial cell production of IL-8, IL6 , and chemokine ligand 20 (CCL20) and promote mucosal t-helper cell (Th17) immune response and neutrophil recruitment. Besides, Prevotella was positively correlated with IL-1 $\beta$, IL-6, and TNF- $a^{59,60}$. Previous studies concluded that dietary RES alleviated gut inflammation and decreased the concentration of IL- 6 and TNF- $\alpha$ in the jejunum of piglets ${ }^{55}$ while we observed that dietary RES supplementation with OSOs decreased the levels of IL-1 $1 \beta, \mathrm{IL}-6, \mathrm{IL}-8$, and TNF- $\alpha$ in the plasma of weaned piglets. In addition, dietary RES supplementation in the diets including OSOs tended to increase the abundance of 
Atopobiaceae_unclassified, which was negatively correlated with IL-6 in the plasma of weaned piglets. Additionally, it was suggested that inhibition of C-C chemokine motif ligand 4 could increase intestinal Atopobiaceae abundance, inhibiting pro-inflammatory metabolites and reducing systemic inflammation ${ }^{61}$. These results demonstrated that RES might exert its anti-inflammatory effects by reducing the abundance of the colonic content species Prevotella-1, Prevotellaceae UCG003, and Atopobiaceae_unclassified to balance the cytokines in the plasma. Interestingly, the abundance of Prevotella_ 1 and Prevotellaceae_UCG-003 were negatively correlated with EE digestion. Meanwhile, the abundance of the former was positively correlated with the change of diarrhea rate from 13 to 16 days, while the latter's abundance was negatively correlated with the change of a-amylase in weaned piglets. It was also proved that hosts with Prevotella as the dominant gut flora were negatively correlated with feed efficiency and weight ${ }^{62,63}$. However, a positive correlation was found between the compromised Prevotella intestinal flora, body weight, and average daily gain of pigs ${ }^{64}$. Therefore, we hypothesized that these conflicting studies might be different with strains associated with specific Prevotella spp. or positive and negative growth outcomes and that the nature of RES seemed to be different sometimes. In addition, we found that the dietary RES supplemented with OSOs failed to influence the abundance of Mollicutes_RF39_unclassified, which only increased when diets were supplemented with FSOs. According to reports, Mollicutes was associated with prostate cancer and bovine respiratory disease ${ }^{65,66}$. Conversely, several scholars reported RES and its analogs as an effective miRNA-mediated chemopreventive and therapeutic strategy in prostate cancer ${ }^{67,68}$. The contradictory results could be related to the dose of RES. RES modulate immune was reported to function in a dose-dependent manner, with low doses stimulating the immune system and high doses inducing immunosuppression, while faulty immune responses played a pathogenic role in a wide range of inflammatory diseases ${ }^{69}$.

Recent years saw the emerging links between early gut flora imbalance and host disease risk. SCFAs were major final products of indigestible carbohydrates produced by microorganisms through fermentation, functioning as ligands for various immune responses, activating/targeting cell receptors expressed on specific host tissues and immune cell membranes ${ }^{70}$. OSOs reduced acetic acid, and dietary RES increased acetic acid and butyric acid levels in the colon of weaned piglets. Past research demonstrated that dietary RES could increase butyric acid and isobutyric acid levels in the intestines of mice ${ }^{71}$, while butyric acid could alleviate gut inflammation by reducing IL- 6 and IL-1 $\beta$ levels and increasing IL-10 levels in broilers ${ }^{72}$. This study discovered that diets including OSOs enhanced the expression of mRNA proinflammatory cytokines such as IL-1 $\beta$ and TNF- $\alpha$ in the jejunum of weaned piglets, consistent with the previous results ${ }^{58}$. Dietary RES decreased TNF- $\alpha$ and NF-KB mRNA expression in the jejunum and TLR4 mRNA expression in the colon. The mRNA expression of IL-1 $\beta$ and TNF-a was directly or indirectly related to the activation of the NF-KB pathway ${ }^{73}$. SCFAs were used as histone deacetylase (HDAC) inhibitors to attenuate inflammatory responses by blocking the NF-kB signaling pathway ${ }^{74,75}$. Therefore, we hypothesized that RES might alleviate gut inflammation caused by OSOs-induced NF-KB pathway by regulating SCFAs. Besides, SCFAs played an important role in gut health, acted as an energy source for the host ${ }^{76}$, and were proved to promote adipocyte differentiation in porcine adipose tissue $\mathrm{e}^{77}$. 
Nevertheless, our study noticed that dietary RES increased the expression of FABP1 in the jejunum and the expression of FABP1 PPARY, and CD36 mRNA in the colon of weaned piglets. FABP1 was significant in the normal lipid metabolism of differentiated gut epithelial cells, especially the uptake and basolateral secretion of fatty acids ${ }^{78}$. Meanwhile, the CD36 receptor, as a multifunctional membrane receptor, effectively promoted the ability of fatty acid uptake and improved lipid metabolism ${ }^{79}$. Furthermore, a clinical study revealed that propionic acid and butyric acid could promote the formation of pig adipocytes

and increase the expression of PPAR- $y$, a transcriptional factor crucial for controlling lipid homeostasis ${ }^{80}$, as well as CEBPA mRNA in a stromal vascular fraction ${ }^{77}$. Our previous study demonstrated that maternal dietary RES increased fat content in longissimus Pectoris and milk ${ }^{36,40}$. The inclusion of RES in the diet was reported to promote the fatty acid oxidation rate and energy release ${ }^{80}$. In addition, SCFAs participated in various host-signaling mechanisms and were crucial in inhibiting histone deacetylases and activating G-coupled-protein receptors ${ }^{81}$. This work observed that RES increased GPR41 protein levels in piglets, which agreed with the previous research results ${ }^{82}$. Therefore, we hypothesized that RES could affect the mRNA expression of FABP1, CD36, and PPARY by increasing the level of short-chain fatty acids, thus alleviating the effect of OSOs on the energy metabolism of weaned piglets.

\section{Conclusions}

In conclusion, dietary RES improved the villus-crypt structure and digestive enzyme activities of jejunum, thus reducing intestinal barrier damage in weaned piglets with diets including OSOs. The dietary RES supplementation increased the antioxidant status in plasma, thereby increasing the antioxidant status of weaned piglets. In addition, RES may alleviate OSOs immune status and energy metabolism by affecting gut microbiota and its metabolite SCFAs. Notably, the positive effect of RES on OSOs was related to the decrease in the abundance of Prevotella_1 and Prevotellaceae_UCG-003.

\section{Abbreviations}

RES: resveratrol; FSOs: fresh soybean oils; OSOs: oxidized soybean oils; ADFI: average daily feed intake; ADG: average daily gain; F/G: average daily feed intake/average daily gain; DM: dry matter; CP: crude protein, EE: crude fat; GE: gross energy; VH: villus height; CD: crypt depth of the jejunum. VCR: villus/crypt ratio; DAO: diamine oxidase; T-SOD: total superoxide dismutase; GSH-Px: glutathione peroxidase; $\mathrm{H} 2 \mathrm{O} 2$ : hydrogen peroxide; T-AOC: total antioxidant capacity; MDA: malondialdehyde; IL-1 $\beta$, interleukin 1 $\beta$; IL-6, interleukin 6; IL-8, interleukin 8; IL-10, interleukin 10; IL-17, interleukin 17; IL-22, interleukin 22; TNF-a, tumor necrosis factor $\alpha$; NF-KB, nuclear factor KB; SCFAs: Short-chain fatty acids; ZO-1: Zonula occludens1; GPR41: G-protein-coupled receptor-41; GPR43: G-protein-coupled receptor-41; PPARy: peroxisome proliferator-activated receptor-y; FABP1: fatty acid-binding protein-1; FABP4: fatty acid-binding protein-4; CD36: fatty acid transport protein.

\section{Declarations}


Acknowledgments

The authors thank all the participants and staff of this trial for their valuable contributions.

\section{Funding}

This work was supported by the National Natural Science Foundation of China (31872986).

\section{Availability of data and material}

The datasets produced and/or analyzed during the current study are available from the corresponding author on reasonable request.

\section{Authors' contributions}

The author contributions are as follows: B. S., Q. M., and Y. G. conceived and designed the experimental plan. Y. G., Q. M., X. S. and Q. Z. were involved in the animal experiments, analysis and data collection.Y. G., Q. M., X. S. and Q. Z. analyzed the data and drafted the original manuscript. X. S. carried out the additional experiment and collected the data. Y. G. and Q. M. made a revision of this manuscript. All authors read and approved the final manuscript.

\section{Competing interests}

None of the authors had any personal or financial conflict of interest.

\section{Consent for publication}

Not applicable.

\section{Ethics approval and consent to participate}

The protocols used in this experiment were approved by the Northeast Agricultural University Institutional Animal Care and Use Committee. All animal experimental procedures were approved by the Ethical and Animal Welfare Committee of Heilongjiang Province, China.

\section{References}

1. Wealleans AL, Bierinckx K and di Benedetto M. Fats and oils in pig nutrition: Factors affecting digestion and utilization. Animal Feed Science and Technology. 2021;277. https://doi.org/10.1016/j.anifeedsci.2021.114950.

2. Kerr BJ, Lindblom SC and Overholt MF. Influence of feeding thermally peroxidized soybean oil on growth performance, digestibility, gut integrity, and oxidative stress in nursery pigs. Journal of Animal Science. 2020;98(2). https://doi.org/10.1093/jas/skaa016. 
3. National Research Council. Nutrient requirements of Swine. National Academy Press, Washington. 2009. $11^{\text {th }}$ edn.

4. Cabel MC, Waldroup PW, Shermer WD and Calabotta DF. Effects of ethoxyquin feed preservative and peroxide level on broiler performance. Poultry Science. 1988;67(12):1725-1730. https://doi.org/10.3382/ps.0671725.

5. Degroote J, Wang W, Vergauwen H, De Smet S, Van Ginneken C and Michiels J. Impact of a dietary challenge with peroxidized oil on the glutathione redox status and integrity of the small intestine in weaned piglets. Animal. 2019;13(8):1641-1650. https://doi.org/10.1017/s1751731118003166.

6. Tavarez MA, Boler DD, Bess KN, Zhao J, Yan F, Dilger AC, et al. Effect of antioxidant inclusion and oil quality on broiler performance, meat quality, and lipid oxidation. Poultry Science. 2011;90(4):922930. https://doi.org/10.3382/ps.2010-01180.

7. Zhou ZK, Wang YY, Jiang YM, Diao YJ, Strappe P, Prenzler P, et al. Deep-fried oil consumption in rats impairs glycerolipid metabolism, gut histology and microbiota structure. Lipids in Health and Disease. 2016;15. https://doi.org/10.1186/s12944-016-0252-1.

8. Arowolo FK, Yang X, Blaser ME, Nicholson AM, Hosokawa E, Booth JR, et al. Presence of lipid oxidation products in swine diet lowers pork quality and stability during storage. Meat Science. 2020;160(https://doi.org/10.1016/j.meatsci.2019.107946.

9. Garcia GR, Dogi CA, Ashworth GE, Berardo D, Godoy G, Cavaglieri LR, et al. Effect of breast feeding time on physiological, immunological and microbial parameters of weaned piglets in an intensive breeding farm. Veterinary Immunology and Immunopathology. 2016;176:44-49. https://doi.org/10.1016/j.vetimm.2016.02.009.

10. Boler DD, Fernandez-Duenas DM, Kutzler LW, Zhao J, Harrell RJ, Campion DR, et al. Effects of oxidized corn oil and a synthetic antioxidant blend on performance, oxidative status of tissues, and fresh meat quality in finishing barrows. Journal of Animal Science. 2012;90(13):5159-5169. https://doi.org/10.2527/jas.2012-5266.

11. Cao ST, Shen ZJ, Wang CC, Zhang QH, Hong QH, He YH, et al. Resveratrol improves intestinal barrier function, alleviates mitochondrial dysfunction and induces mitophagy in diquat challenged piglets(1). Food \& Function. 2019;10(1):344-354. https://doi.org/10.1039/c8fo02091d.

12. Ge YT, Liu W, Tao HT, Zhang Y, Liu LN, Liu ZH, et al. Effect of industrial trans-fatty acids-enriched diet on gut microbiota of c57bl/6 mice. European Journal of Nutrition. 2019;58(7):2625-2638. https://doi.org/10.1007/s00394-018-1810-2.

13. Gresse R, Chaucheyras-Durand F, Fleury MA, Van de Wiele T, Forano E and Blanquet-Diot S. Gut microbiota dysbiosis in postweaning piglets: Understanding the keys to health. Trends in Microbiology. 2017;25(10):851-873. https://doi.org/10.1016/j.tim.2017.05.004.

14. Yan HL, Zhou P, Zhang Y, Zhang ZZ, Liu JB and Zhang HF. Short-chain fructo-oligosaccharides alleviates oxidized oil-induced intestinal dysfunction in piglets associated with the modulation of gut microbiota. Journal of Functional Foods. 2020;64. https://doi.org/10.1016/j.jff.2019.103661. 
15. Zhang H, Chen YN, Chen YP, Ji SL, Jia PL, Li Y, et al. Comparison of the protective effects of resveratrol and pterostilbene against intestinal damage and redox imbalance in weanling piglets. Journal of Animal Science and Biotechnology. 2020;11(1). https://doi.org/10.1186/s40104-02000460-3.

16. Wang DF, Zhou LL, Zhou HL, Hu HC and Hou GY. Chemical composition and protective effect of guava (psidium guajava I.) leaf extract on piglet intestines. Journal of the Science of Food and Agriculture. 2021;101(7):2767-2778. https://doi.org/10.1002/jsfa.10904.

17. Zhang C, Luo JQ, Yu B, Zheng P, Huang ZQ, Mao XB, et al. Dietary resveratrol supplementation improves meat quality of finishing pigs through changing muscle fiber characteristics and antioxidative status. Meat Science. 2015;102:15-21. https://doi.org/10.1016/j.meatsci.2014.11.014.

18. Chen XL, Zeng ZY, Huang ZQ, Chen DW, He J, Chen H, et al. Effects of dietary resveratrol supplementation on immunity, antioxidative capacity and intestinal barrier function in weaning piglets. Animal Biotechnology. 2021;32(2):240-245.

https://doi.org/10.1080/10495398.2019.1683022.

19. Meng QW, Guo T, Li GQ, Sun SS, He SQ, Cheng BJ, et al. Dietary resveratrol improves antioxidant status of sows and piglets and regulates antioxidant gene expression in placenta by keap1-nrf2 pathway and sirt1. Journal of Animal Science and Biotechnology. 2018;9. https://doi.org/10.1186/s40104-018-0248-y.

20. Gan ZD, Wei WY, Li Y, Wu JM, Zhao YW, Zhang LL, et al. Curcumin and resveratrol regulate intestinal bacteria and alleviate intestinal inflammation in weaned piglets. Molecules. 2019;24(7). https://doi.org/10.3390/molecules24071220.

21. Fu QY, Tan Z, Shi LG and Xun WJ. Resveratrol attenuates diquat-induced oxidative stress by regulating gut microbiota and metabolome characteristics in piglets. Frontiers in Microbiology. 2021;12. https://doi.org/10.3389/fmicb.2021.695155.

22. D. Firestone, A. O. C. Society, 2009.

23. Liu P, Piao XS, Thacker PA, Zeng ZK, Li PF, Wang D, et al. Chito-oligosaccharide reduces diarrhea incidence and attenuates the immune response of weaned pigs challenged with escherichia coli k88. Journal of Animal Science. 2010;88(12):3871-3879. https://doi.org/10.2527/jas.2009-2771.

24. Ren P, Zhu ZP, Dong B, Zang JJ and Gong LM. Determination of energy and amino acid digestibility in growing pigs fed corn distillers' dried grains with solubles containing different lipid levels. Archives of Animal Nutrition. 2011;65(4):303-319. https://doi.org/10.1080/1745039x.2011.588849.

25. Teng T, Gao F, He W, Fu HY, Guo J, Bai GD, et al. An early fecal microbiota transfer improves the intestinal conditions on microflora and immunoglobulin and antimicrobial peptides in piglets. Journal of Agricultural and Food Chemistry. 2020;68(17):4830-4843. https://doi.org/10.1021/acs.jafc.0c00545.

26. Livak KJ and Schmittgen TD. Analysis of relative gene expression data using real-time quantitative pcr and the 2(t)(-delta delta c) method. Methods. 2001;25(4):402-408. https://doi.org/10.1006/meth.2001.1262. 
27. Caspary WF. Physiology and pathophysiology of intestinal-absorption. American Journal of Clinical Nutrition. 1992;55(1):299-308. https://doi.org/10.1093/ajcn/55.1.299s.

28. Fukudome I, Kobayashi M, Dabanaka K, Maeda H, Okamoto K, Okabayashi T, et al. Diamine oxidase as a marker of intestinal mucosal injury and the effect of soluble dietary fiber on gastrointestinal tract toxicity after intravenous 5-fluorouracil treatment in rats. Medical Molecular Morphology. 2014;47(2):100-107. https://doi.org/10.1007/s00795-013-0055-7.

29. Szalay L, Umar F, Khadem A, Jafarmadar M, Furst W, Ohlinger W, et al. Increased plasma d-lactate is associated with the severity of hemorrhagic/traumatic shock in rats. Shock. 2003;20(3):245-250. https://doi.org/10.1097/00024382-200309000-00008.

30. Cherbuy C, Andrieux C, Honvo-Houeto E, Thomas M, Ide C, Druesne N, et al. Expression of mitochondrial hmgcoa synthase and glutaminase in the colonic mucosa is modulated by bacterial species. European Journal of Biochemistry. 2004;271(1):87-95. https://doi.org/10.1046/j.14321033.2003.03908.x.

31. Frese SA, Parker K, Calvert CC and Mills DA. Diet shapes the gut microbiome of pigs during nursing and weaning. Microbiome. 2015;3. https://doi.org/10.1186/s40168-015-0091-8.

32. Hampson DJ. Alterations in piglet small intestinal structure at weaning. Research in Veterinary Science. 1986;40(1):32-40. https://doi.org/10.1016/s0034-5288(18)30482-x.

33. Overholt MF, Dilger AC, Boler DD and Kerr BJ. Influence of feeding thermally peroxidized soybean oil on growth performance, digestibility, and gut integrity in finishing pigs. Journal of Animal Science. 2018;96(7):2789-2803. https://doi.org/10.1093/jas/sky091.

34. Zhang C, Zhao XH, Yang L, Chen XY, Jiang RS, Jin SH, et al. Resveratrol alleviates heat stressinduced impairment of intestinal morphology, microflora, and barrier integrity in broilers. Poultry Science. 2017;96(12):4325-4332. https://doi.org/10.3382/ps/pex266.

35. Liu P, Kerr BJ, Weber TE, Chen C, Johnston LJ and Shurson GC. Influence of thermally oxidized vegetable oils and animal fats on intestinal barrier function and immune variables in young pigs. Journal of Animal Science. 2014;92(7):2971-2979. https://doi.org/10.2527/jas.2012-5710.

36. Sun SS, Meng QW, Luo Z, Shi BM, Bi CP and Shan AS. Effects of dietary resveratrol supplementation during gestation and lactation of sows on milk composition of sows and fat metabolism of sucking piglets. Journal of Animal Physiology and Animal Nutrition. 2019;103(3):813-821. https://doi.org/10.1111/jpn.13064.

37. Cheng K, Song ZH, Zhang H, Li SM, Wang C, Zhang LL, et al. The therapeutic effects of resveratrol on hepatic steatosis in high-fat diet-induced obese mice by improving oxidative stress, inflammation and lipid-related gene transcriptional expression. Medical Molecular Morphology. 2019;52(4):187197. https://doi.org/10.1007/s00795-019-00216-7.

38. Zhang YW, Luo HL, Chang YF, Jiao LJ and Liu K. Effects of liquorice extract on the activity and gene expression level of antioxidant enzymes in longissimus dorsi muscle of tan lamb. Small Ruminant Research. 2017;154:23-28. https://doi.org/10.1016/j.smallrumres.2017.06.012. 
39. Vega CC, Reyes-Castro LA, Rodriguez-Gonzalez GL, Bautista CJ, Vazquez-Martinez M, Larrea F, et al. Resveratrol partially prevents oxidative stress and metabolic dysfunction in pregnant rats fed a low protein diet and their offspring. Journal of Physiology-London. 2016;594(5):1483-1499. https://doi.org/10.1113/jp271543.

40. Meng QW, Sun SS, Bai YS, Luo Z, Li ZY, Shi BM, et al. Effects of dietary resveratrol supplementation in sows on antioxidative status, myofiber characteristic and meat quality of offspring. Meat Science. 2020;167. https://doi.org/10.1016/j.meatsci.2020.108176.

41. Wang N, Han Q, Wang G, Ma WP, Wang J, Wu WX, et al. Resveratrol protects oxidative stress-induced intestinal epithelial barrier dysfunction by upregulating heme oxygenase-1 expression. Digestive Diseases and Sciences. 2016;61(9):2522-2534. https://doi.org/10.1007/s10620-016-4184-4.

42. Zihni $\mathrm{C}$, Mills $\mathrm{C}$, Matter $\mathrm{K}$ and Balda MS. Tight junctions: From simple barriers to multifunctional molecular gates. Nature Reviews Molecular Cell Biology. 2016;17(9):564-580. https://doi.org/10.1038/nrm.2016.80.

43. Vilar E, Salazar R, Perez-Garcia J, Cortes J, Oberg K and Tabernero J. Chemotherapy and role of the proliferation marker ki-67 in digestive neuroendocrine tumors. Endocrine-Related Cancer. 2007;14(2):221-232. https://doi.org/10.1677/erc-06-0074.

44. Zhang DY, Ji HF, Liu H, Wang SX, Wang J and Wang YM. Changes in the diversity and composition of gut microbiota of weaned piglets after oral administration of lactobacillus or an antibiotic. Applied Microbiology and Biotechnology. 2016;100(23):10081-10093. https://doi.org/10.1007/s00253-0167845-5.

45. Thomas F, Hehemann JH, Rebuffet E, Czjzek M and Michel G. Environmental and gut bacteroidetes: The food connection. Frontiers in Microbiology. 2011;2. https://doi.org/10.3389/fmicb.2011.00093.

46. Ley RE, Turnbaugh PJ, Klein S and Gordon JI. Microbial ecology - human gut microbes associated with obesity. Nature. 2006;444(7122):1022-1023. https://doi.org/10.1038/4441022a.

47. Lim MY, You HJ, Yoon HS, Kwon B, Lee JY, Lee S, et al. The effect of heritability and host genetics on the gut microbiota and metabolic syndrome. Gut. 2017;66(6):1031-1038. https://doi.org/10.1136/gutjnl-2015-311326.

48. Ushiroda C, Naito Y, Takagi T, Uchiyama K, Mizushima K, Higashimura Y, et al. Green tea polyphenol (epigallocatechin-3-gallate) improves gut dysbiosis and serum bile acids dysregulation in high-fat diet-fed mice. Journal of Clinical Biochemistry and Nutrition. 2019;65(1):34-46. https://doi.org/10.3164/jcbn.18-116.

49. Niu Q, Li PH, Hao SS, Zhang YQ, Kim SW, Li HZ, et al. Dynamic distribution of the gut microbiota and the relationship with apparent crude fiber digestibility and growth stages in pigs. Scientific Reports. 2015;5. https://doi.org/10.1038/srep09938.

50. Zhou J, Xiong X, Wang KX, Zou LJ, Ji P and Yin YL. Ethanolamine enhances intestinal functions by altering gut microbiome and mucosal anti-stress capacity in weaned rats. British Journal of Nutrition. 2018;120(3):241-249. https://doi.org/10.1017/s0007114518001101. 
51. Harlow BE, Lawrence LM and Flythe MD. Diarrhea-associated pathogens, lactobacilli and cellulolytic bacteria in equine feces: Responses to antibiotic challenge. Veterinary Microbiology. 2013;166(12):225-232. https://doi.org/10.1016/j.vetmic.2013.05.003.

52. Saltiel E. Clostridium difficile-associated diarrhea:Role of the pharmacist in the health system. 2013;26(5):462-463. https://doi.org/10.1177/0897190013499520.

53. Wang K, Liao MF, Zhou N, Bao L, Ma K, Zheng ZY, et al. Parabacteroides distasonis alleviates obesity and metabolic dysfunctions via production of succinate and secondary bile acids. Cell Reports. 2019;26(1):222-+. https://doi.org/10.1016/j.celrep.2018.12.028.

54. Overland M, Rorvik KA and Skrede A. Effect of trimethylamine performance, oxide and betaine in swine diets on growth carcass characteristics, nutrient digestibility, and sensory quality of pork. Journal of Animal Science. 1999;77(8):2143-2153. https://doi.org/.

55. Meng QW, Sun SS, Luo Z, Shi BM, Shan AS and Cheng BJ. Maternal dietary resveratrol alleviates weaning-associated diarrhea and intestinal inflammation in pig offspring by changing intestinal gene expression and microbiota. Food \& Function. 2019;10(9):5626-5643. https://doi.org/10.1039/c9fo00637k.

56. Edwards JE, McEwan NR, Travis AJ and Wallace RJ. 16s rdna library-based analysis of ruminal bacterial diversity. Antonie Van Leeuwenhoek International Journal of General and Molecular Microbiology. 2004;86(3):263-281. https://doi.org/10.1023/b:Anto.0000047942.69033.24.

57. Matsui H, Ogata K, Tajima K, Nakamura M, Nagamine T, Aminov RI, et al. Phenotypic characterization of polysaccharidases produced by four prevotella type strains. Current Microbiology. 2000;41(1):4549. https://doi.org/10.1007/s002840010089.

58. Huang L, Ma XY, Jiang ZY, Hu YJ, Zheng CT, Yang XF, et al. Effects of soybean isoflavone on intestinal antioxidant capacity and cytokines in young piglets fed oxidized fish oil. Journal of Zhejiang University-Science B. 2016;17(12):965-974. https://doi.org/10.1631/jzus.B1600078.

59. Scher JU, Sczesnak A, Longman RS, Segata N, Ubeda C, Bielski C, et al. Expansion of intestinal prevotella copri correlates with enhanced susceptibility to arthritis. Elife. 2013;2. https://doi.org/10.7554/eLife.01202.

60. Li RN, Li LQ, Hong P, Lang WY, Hui JN, Yang Y, et al. Beta-carotene prevents weaning-induced intestinal inflammation by modulating gut microbiota in piglets. Animal Bioscience. 2021;34(7):1221-1234. https://doi.org/10.5713/ajas.19.0499.

61. Chang TT and Chen JW. Direct ccl4 inhibition modulates gut microbiota, reduces circulating trimethylamine n-oxide, and improves glucose and lipid metabolism in high-fat-diet-induced diabetes mellitus. Journal of Inflammation Research. 2021;14:6237-6250. https://doi.org/10.2147/jir.S343491.

62. Tan Z, Yang T, Wang Y, Xing K, Zhang FX, Zhao XT, et al. Metagenomic analysis of cecal microbiome identified microbiota and functional capacities associated with feed efficiency in landrace finishing pigs. Frontiers in Microbiology. 2017;8. https://doi.org/10.3389/fmicb.2017.01546. 
63. Unno T, Choi JH, Hur HG, Sadowsky MJ, Ahn YT, Huh CS, et al. Changes in human gut microbiota influenced by probiotic fermented milk ingestion. Journal of Dairy Science. 2015;98(6):3568-3576. https://doi.org/10.3168/jds.2014-8943.

64. Ramayo-Caldas Y, Mach N, Lepage P, Levenez F, Denis C, Lemonnier G, et al. Phylogenetic network analysis applied to pig gut microbiota identifies an ecosystem structure linked with growth traits. Isme Journal. 2016;10(12):2973-2977. https://doi.org/10.1038/ismej.2016.77.

65. Abdul-Wahab OMS, Al-Shyarba MH, Mardassi BB, Sassi N, Al Fayi MSS, Otifi H, et al. Molecular detection of urogenital mollicutes in patients with invasive malignant prostate tumor. Infectious Agents and Cancer. 2021;16(1). https://doi.org/10.1186/s13027-021-00344-9.

66. Tortorelli G, Gaeta NC, Ribeiro BLM, Marques LM, Timenetsky J and Gregory L. Evaluation of mollicutes microorganisms in respiratory disease of cattle and their relationship to clinical signs. Journal of Veterinary Internal Medicine. 2017;31(4):1215-1220. https://doi.org/10.1111/jvim.14721.

67. Kumar A, Rimando AM and Levenson AS. Resveratrol and pterostilbene as a microrna-mediated chemopreventive and therapeutic strategy in prostate cancer. Annals of the New York Academy of Sciences. 2017;1403(1):15-26. https://doi.org/10.1111/nyas.13372.

68. Zaffaroni N and Beretta GL. Resveratrol and prostate cancer: The power of phytochemicals. Current Medicinal Chemistry. 2021;28(24):4845-4862. https://doi.org/10.2174/0929867328666201228124038.

69. Malaguarnera L. Influence of resveratrol on the immune response. Nutrients. 2019;11(5). https://doi.org/10.3390/nu11050946.

70. Hsu CN, Hou CY, Chang-Chien GP, Lin SF, Yang HW and Tain YL. Perinatal resveratrol therapy prevents hypertension programmed by maternal chronic kidney disease in adult male offspring: Implications of the gut microbiome and their metabolites. Biomedicines. 2020;8(12). https://doi.org/10.3390/biomedicines8120567.

71. Alrafas HR, Busbee PB, Chitrala KN, Nagarkatti M and Nagarkatti P. Alterations in the gut microbiome and suppression of histone deacetylases by resveratrol are associated with attenuation of colonic inflammation and protection against colorectal cancer. Journal of Clinical Medicine. 2020;9(6). https://doi.org/10.3390/jcm9061796.

72. Zou X, Ji J, Qu H, Wang J, Shu DM, Wang Y, et al. Effects of sodium butyrate on intestinal health and gut microbiota composition during intestinal inflammation progression in broilers. Poultry Science. 2019;98(10):4449-4456. https://doi.org/10.3382/ps/pez279.

73. Liu T, Zhang LY, Joo D and Sun SC. Nf-kappa b signaling in inflammation. Signal Transduction and Targeted Therapy. 2017;2. https://doi.org/10.1038/sigtrans.2017.23.

74. Zhang L, Jin SW, Wang CD, Jiang R and Wan JY. Histone deacetylase inhibitors attenuate acute lung injury during cecal ligation and puncture-induced polymicrobial sepsis. World Journal of Surgery. 2010;34(7):1676-1683. https://doi.org/10.1007/s00268-010-0493-5.

75. Bernhard D, Tinhofer I, Tonko M, Hubl H, Ausserlechner MJ, Greil R, et al. Resveratrol causes arrest in the s-phase prior to fas-independent apoptosis in cem-c7h2 acute leukemia cells. Cell Death and 
Differentiation. 2000;7(9):834-842. https://doi.org/10.1038/sj.cdd.4400719.

76. Yoshikawa S, Araoka R, Kajihara Y, Ito T, Miyamoto H and Kodama H. Valerate production by megasphaera elsdenii isolated from pig feces. Journal of Bioscience and Bioengineering. 2018;125(5):519-524. https://doi.org/10.1016/j.jbiosc.2017.12.016.

77. Li GL, Yao W and Jiang HL. Short-chain fatty acids enhance adipocyte differentiation in the stromal vascular fraction of porcine adipose tissue. Journal of Nutrition. 2014;144(12):1887-1895. https://doi.org/10.3945/jn.114.198531.

78. Shi LH, Balakrishnan K, Thiagarajah K, Mohd Ismail NI and Yin OS. Beneficial properties of probiotics. Trop Life Sci Res. 2016;27(2):73-90. https://doi.org/10.21315/tlsr2016.27.2.6.

79. Danielewski M, Matuszewska A, Szelag A and Sozanski T. The impact of anthocyanins and iridoids on transcription factors crucial for lipid and cholesterol homeostasis. International Journal of Molecular Sciences. 2021;22(11). https://doi.org/10.3390/ijms22116074.

80. Faghihzadeh F, Hekmatdoost A and Adibi P. Resveratrol and liver: A systematic review. Journal of Research in Medical Sciences. 2015;20(8):797-810. https://doi.org/10.4103/1735-1995.168405.

81. Yoo JY, Groer M, Dutra SVO, Sarkar A and McSkimming DI. Gut microbiota and immune system interactions. Microorganisms. 2020;8(10). https://doi.org/10.3390/microorganisms8101587.

82. Cal C, Garban H, Jazirehi A, Yeh C, Mizutani Y and Bonavida B. Resveratrol and Cancer:

Chemoprevention, Apoptosis, and Chemoimmunosensitizing Activities. Current Medicinal Chemistry Anti-Cancer Agents. 2003;3(2). https://doi.org/10.2174/1568011033353443.

\section{Figures}
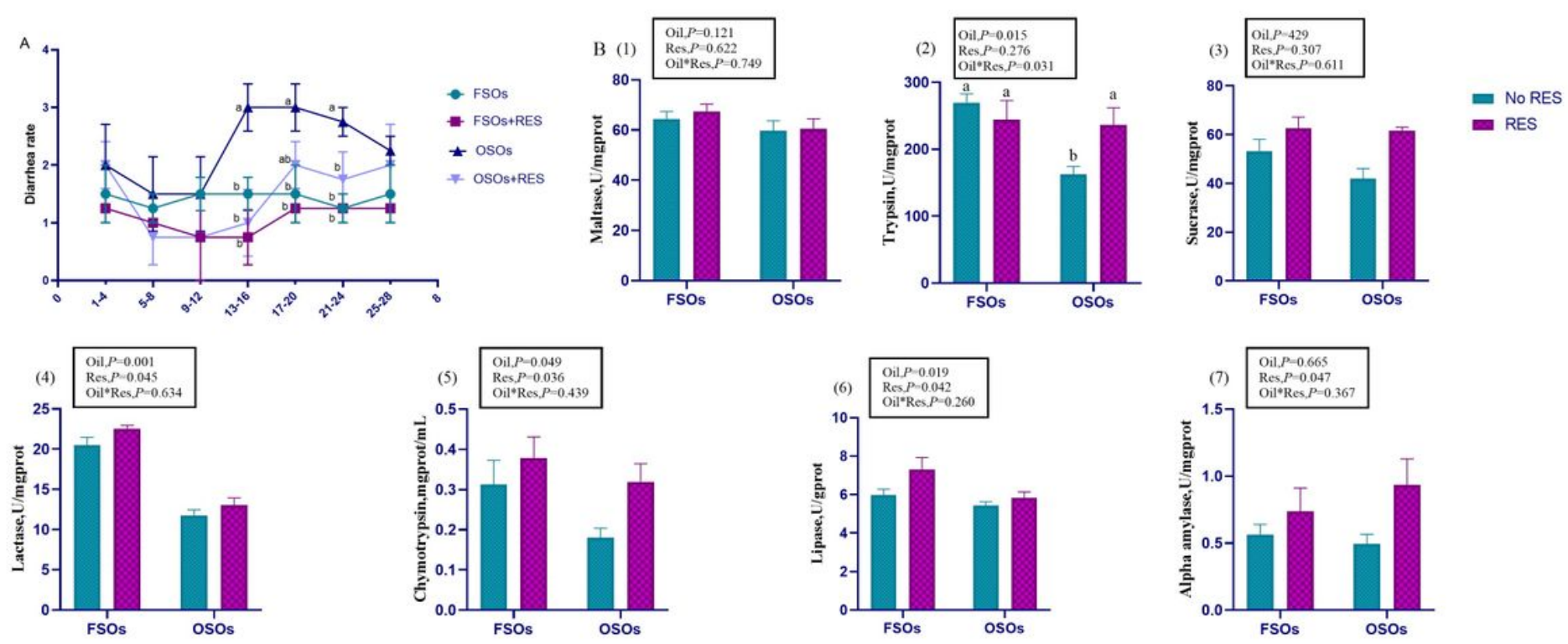

Figure 1 
Effects of OSOs and dietary RES on fecal score and digestive enzyme activity in the jejunum of weaned piglets. (A) Fecal score. (B-1) The activity of maltase in the jejunum. (B-2) The activity of trypsin in the jejunum. (B-3) The activity of sucrase in the jejunum. (B-4) The activity of lactase in the jejunum. (B-5) The activity of chymotrypsin in the jejunum. (B-6) The activity of lipase in the jejunum. (B-7) The activity of alpha-amylase in the jejunum. Note: FSOs, fresh soybean oils; FSOs+ RES, fresh soybean oils with resveratrol; OSOs, oxidized soybean oils; OSOs+ RES, oxidized soybean oils with resveratrol. ${ }^{*} P<0.05$. Oil*RES, interaction between oxidized soybean oils and RES. The column and its bar represent the mean value and standard error ( $n=7$ piglets/group), respectively.
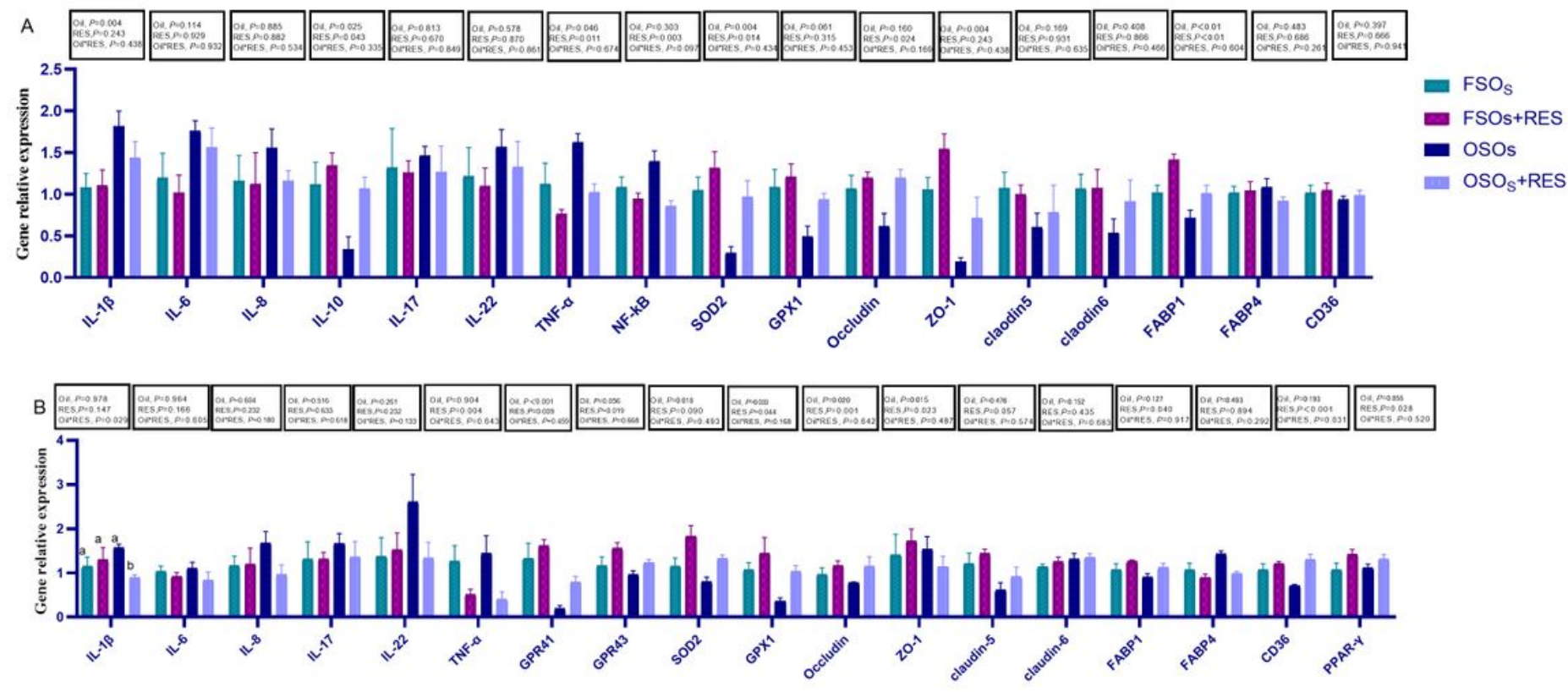

Figure 2

Effects of OSOs and dietary RES on mRNA expression of oxidative stress genes, fat transporter genes and pro-inflammatory cytokines in jejunum and colon of weaned piglets. (A) The mRNA expression of jejunum; (B) The mRNA expression of colon. IL-1 $\beta$, interleukin 1 $\beta$; IL-6, interleukin 6; IL-8, interleukin 8; IL10, interleukin 10; IL-17, interleukin 17; IL-22, interleukin 22; TNF-a, tumor necrosis factor a; NF-KB, nuclear factor KB; SOD2, superoxide dismutase 2; GPX1, glutathione peroxidase 1; ZO-1, Zonula Occludens1;GPR41, G-protein-coupled receptor-41;GPR43, G-protein-coupled receptor-41; PPARy, peroxisome proliferator-activated receptor-ץ; FABP1, fatty acid-binding protein-1; FABP4, fatty acid-binding protein-4; CD36, fatty acid transport protein. The average of $\beta$-actin and GAPDH was used as the internal control. Note: FSOs, fresh soybean oils; FSOs+ RES, fresh soybean oils with resveratrol; OSOs, oxidized soybean oils; OSOs+ RES, oxidized soybean oils with resveratrol. ${ }^{\star} P<0.05$. The column and its bar represent the mean value and standard error ( $n=7$ piglets/group), respectively. 


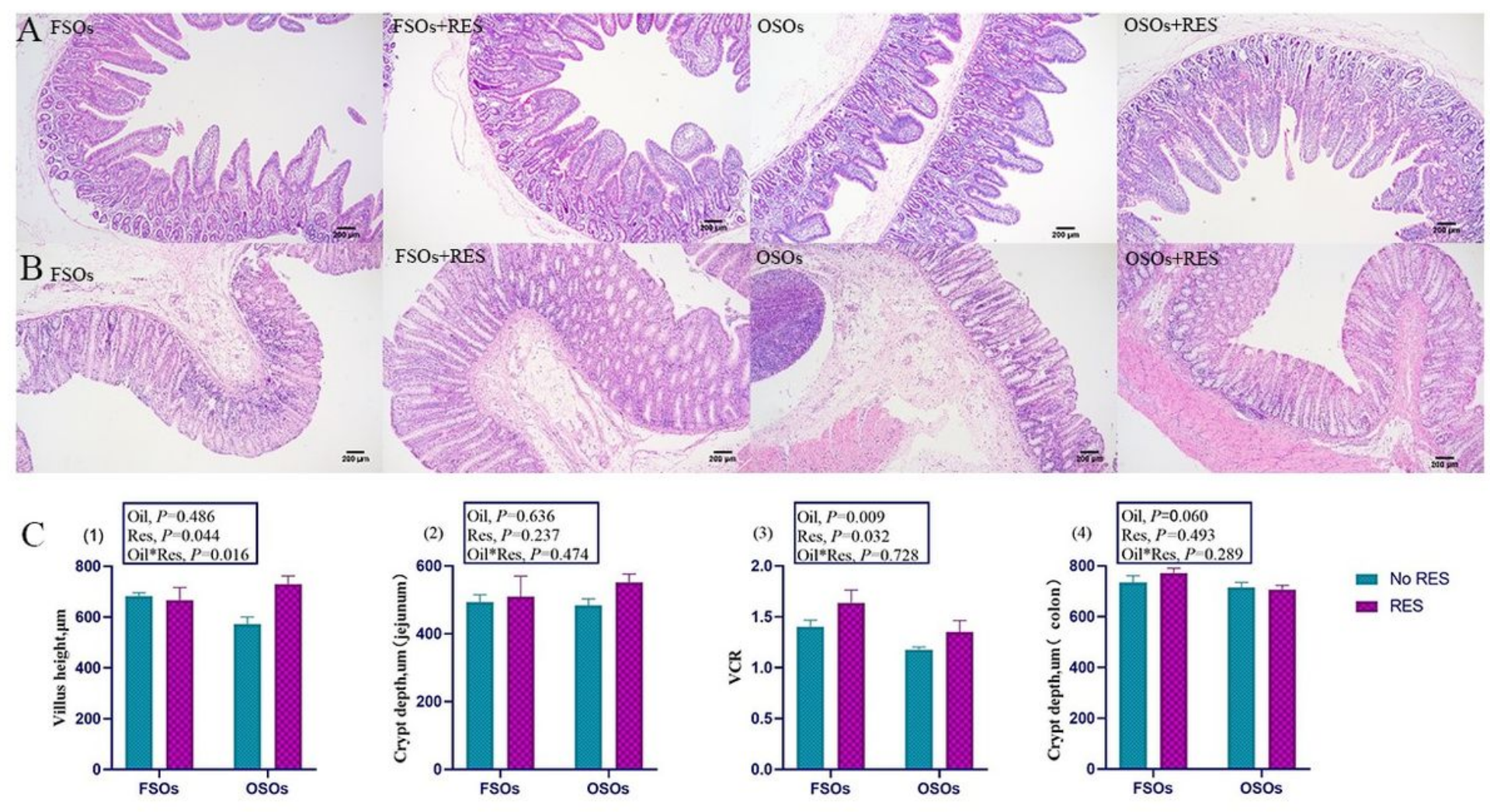

Figure 3

Effects of OSOs and dietary RES on the intestinal morphology in the jejunum and colon of weaned piglets. (A) The H\&E staining of the jejunum. (B) The H\&E staining of the colon. (C-1) Villus height of the jejunum. (C-2) Crypt depth of the jejunum. (C-3) Villus/crypt ratio of the jejunum. (C-4) Crypt depth of the colon. Note: FSOs, fresh soybean oils; FSOs+ RES, fresh soybean oils with resveratrol; OSOs, oxidized soybean oils; OSOs+ RES, oxidized soybean oils with resveratrol. ${ }^{\star} P<0.05$. Oil*RES, interaction between oxidized soybean oils and RES. The column and its bar represent the mean value and standard error $(n=7$ piglets/group), respectively. 
A
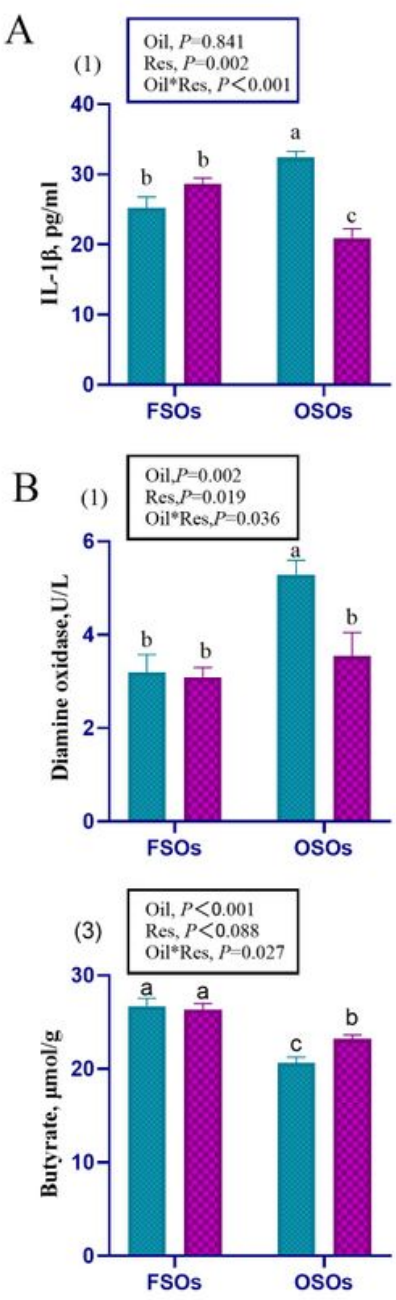
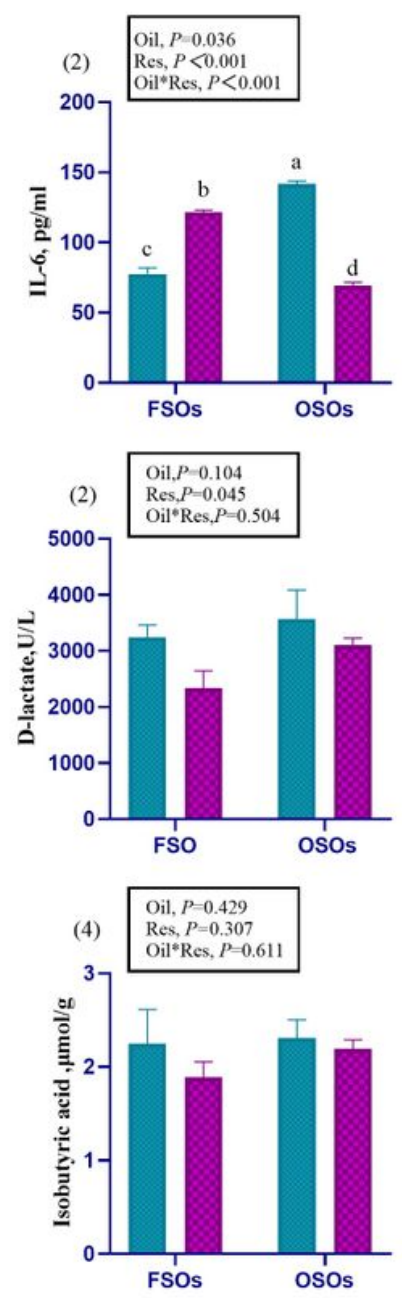
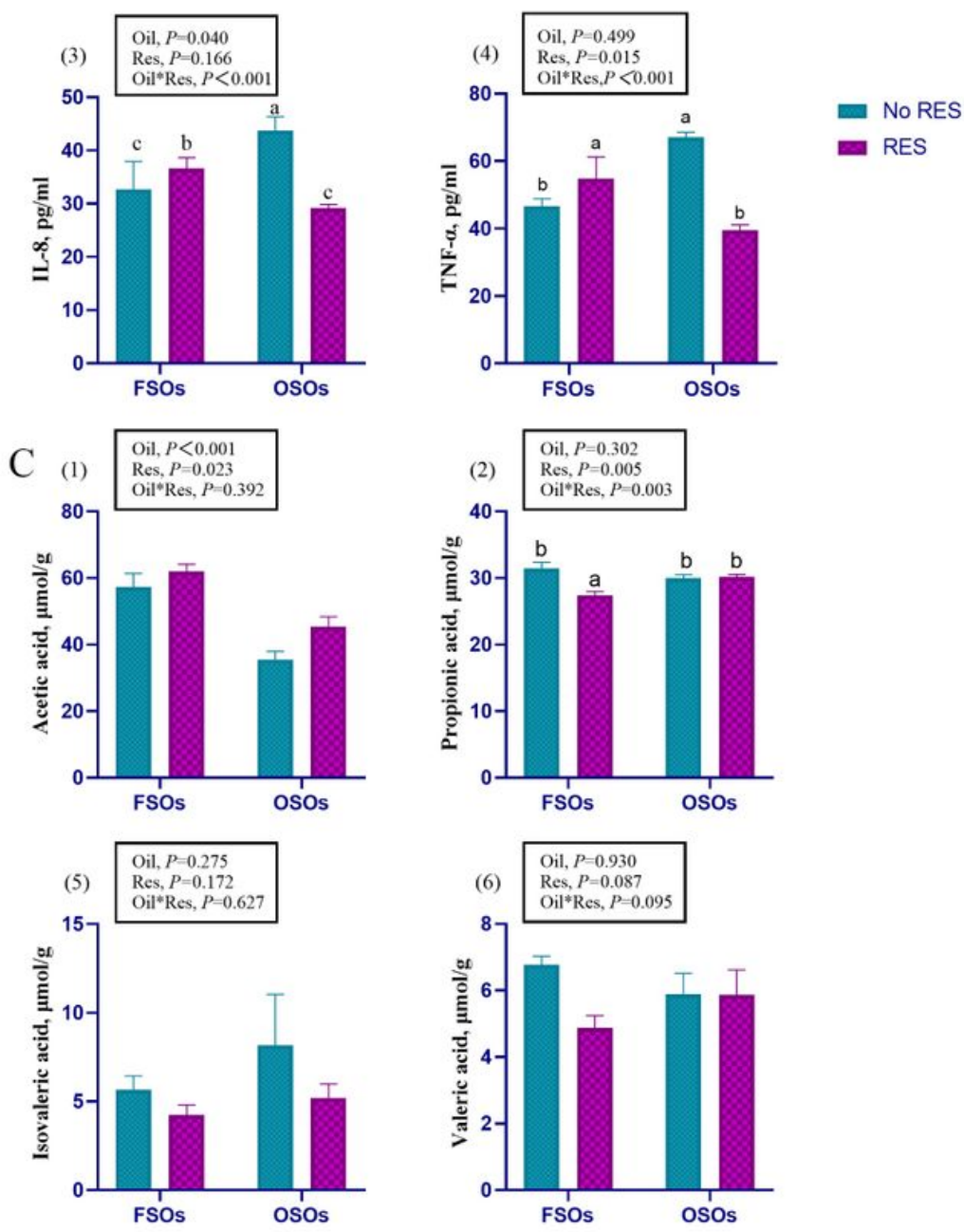

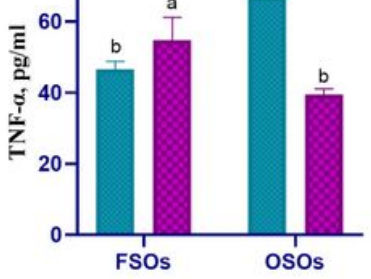

. RES
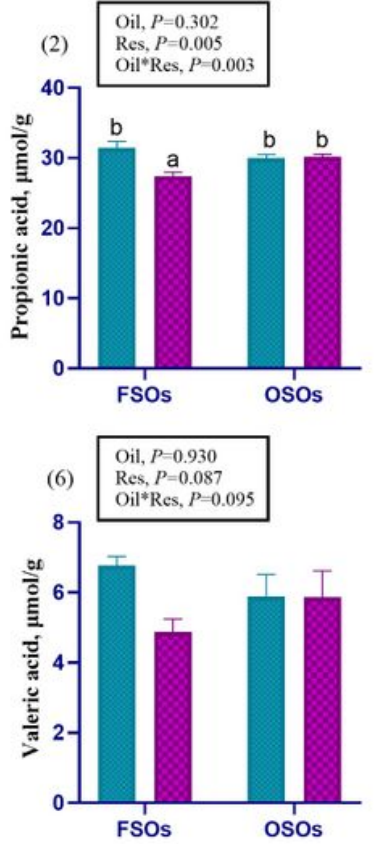

Figure 4

Effects of OSOs and dietary RES on the inflammatory factors, DAO activity, D-lactic and SCFAs content of weaned piglets. (A-1) IL-1 $\beta$, interleukin 1 $\beta$; (A-2) IL-6, interleukin 6; (A-3) IL-8, interleukin 8; (A-4) TNF-a, tumor necrosis factor a. (B-1) DAO, the activity of diamine oxidase in the plasma; (B-2) D-lactic, the content of D-lactic in the plasma; (C-1) The level of acetic acid in the jejunum; (C-2) The level of propionic acid in the jejunum; (C-3) The level of butyric acid in the jejunum; (C-4) The level of isobutyric acid in the jejunum; (C-5) The level of isovaleric acid in the jejunum; (C-6) The level of valeric acid in the jejunum. Note: FSOs, fresh soybean oils; FSOs+ RES, fresh soybean oils with resveratrol; OSOs, oxidized soybean oils; OSOs+ RES, oxidized soybean oils with resveratrol. ${ }^{\star} P<0.05$. Oil*RES, interaction between oxidized soybean oils and RES. All values are expressed as the means \pm SEM. Different letters indicate that the change between each group is statistically significant $(P<0.05)$. The column and its bar represent the mean value and standard error ( $n=7$ piglets/group), respectively. 


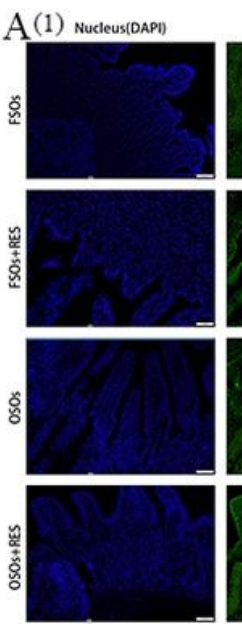

$\mathrm{B}^{(1)}$ Nucleus(DAPI)
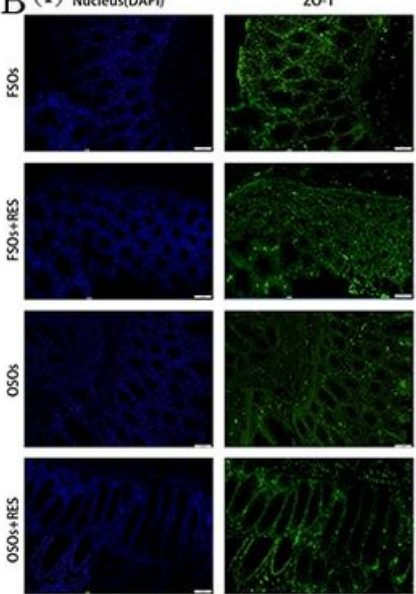

C
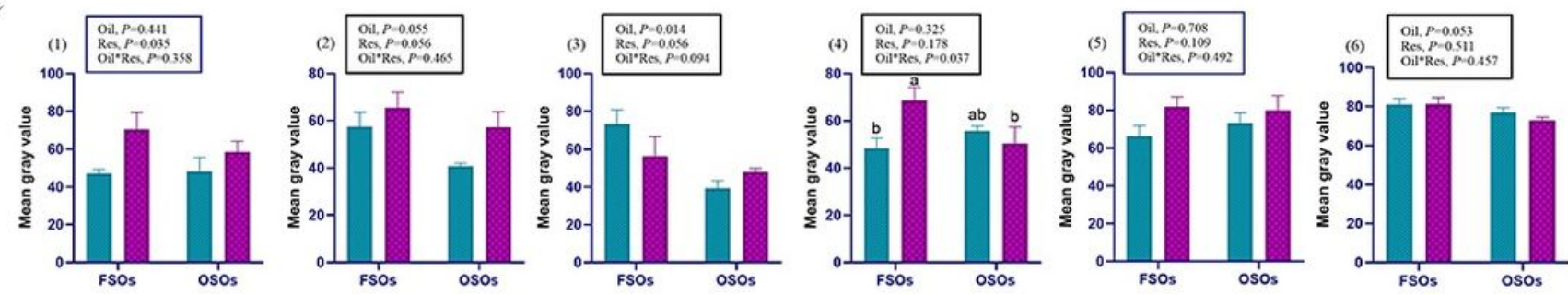

NoRES DES
Ki-67
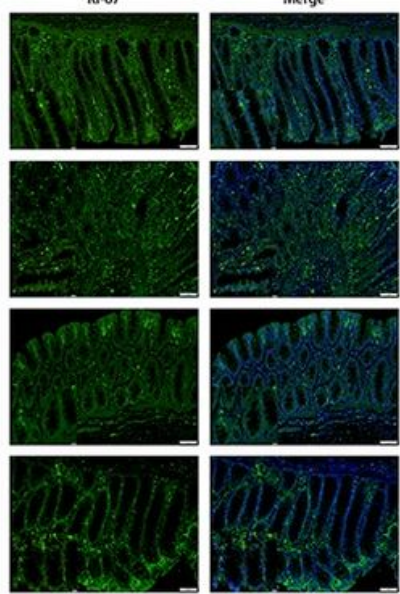

\section{Figure 5}

ZO-1, Occludin, Ki-67 staining on immunofluorescence images in paraformaldehyde-fixed cross-sections from the jejunum and colon of weaned piglets. (100 $\times$ and $400 \times$ magnification), (scale bar, $200 \mu m)(A-1)$ Tight junction protein 1 staining on immunofluorescence images in the jejunum. (A-2) Occludin staining on immunofluorescence images in the jejunum; (A-3) Ki-67 staining on immunofluorescence images in the jejunum. (B-1) Tight junction protein 1 staining on immunofluorescence images in the colon; (B-2) Occludin staining on immunofluorescence images in the colon; (B-3) Ki-67 staining on immunofluorescence images in the colon. (C-1) The relative amount immunofluorescence quantification of ZO-1 in the jejunum samples. (C-2) The relative amount immunofluorescence quantification of Occludin in the jejunum samples. (C-3) The relative amount immunofluorescence quantification of Ki67 in the jejunum samples. (C-4) The relative amount immunofluorescence quantification of ZO-1 in the 
colon samples (C-5) The relative amount immunofluorescence quantification of Occludin in the colon samples. (C-6) The relative amount immunofluorescence quantification of Ki67 in the colon samples. Note: FSOs, fresh soybean oils; FSOs+ RES, fresh soybean oils with resveratrol; OSOs, oxidized soybean oils; OSOs+ RES, oxidized soybean oils with resveratrol. ${ }^{*} P<0.05$. Oil*RES, interaction between oxidized soybean oils and RES. The column and its bar represent the mean value and standard error $(n=7$ piglets/group), respectively.
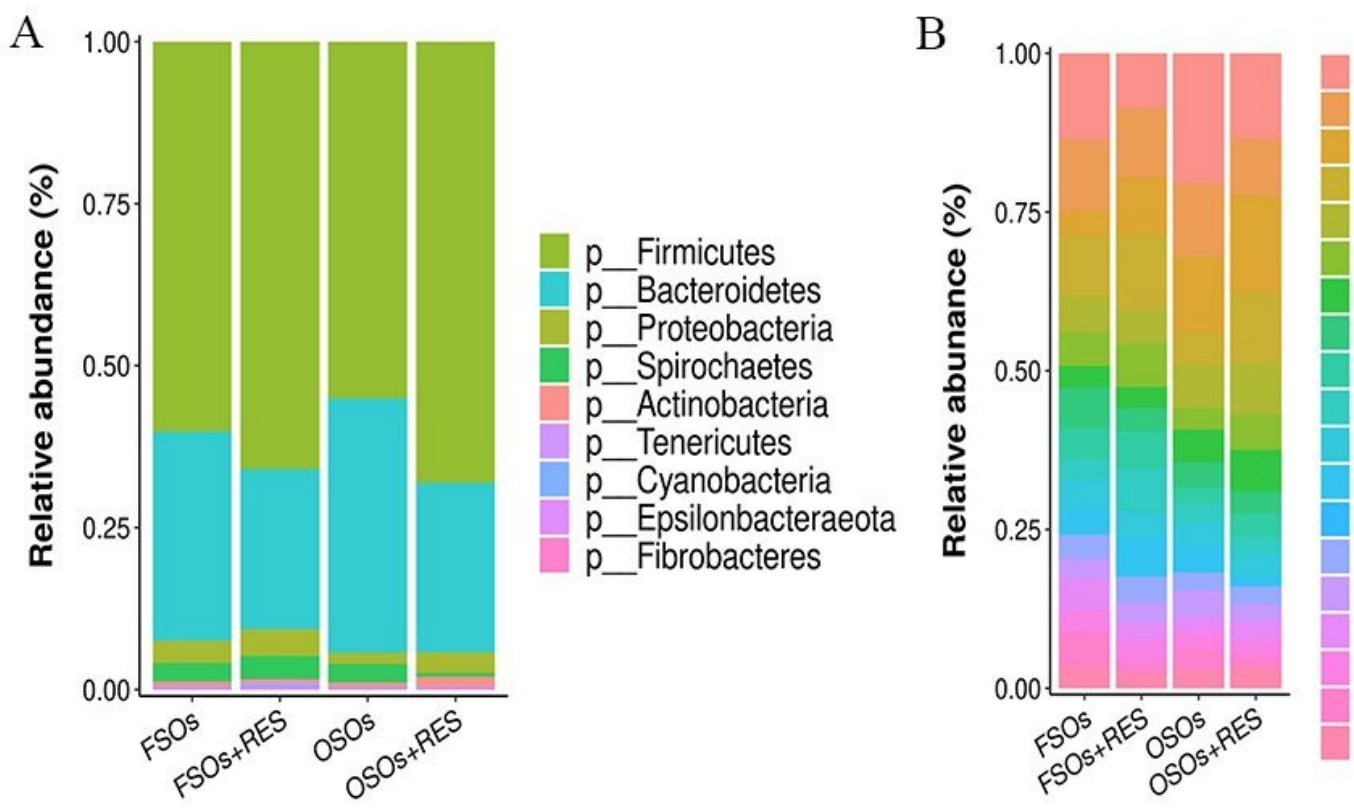

g_Prevotella_9

g__Muribaculaceae_unclassified

g__ Megasphaera

g_Selenomonas

g_Lactobacillus

g_Ruminococcaceae_UCG-005

g_Streptococcus

g_Lachnospiraceae_unclassified

g_Ruminococcaceae_NK4A214_group

g_Prevotellaceae_NK3B31_group

g_Prevotella

g_Treponema_2

g_Veillonella

g_Rikenellaceae_RC9_gut_group

g_Anaerovibrio

g_Agathobacter

g_Alloprevotella

g_Prevotella_2

g_Phascolarctobacterium

\section{C}
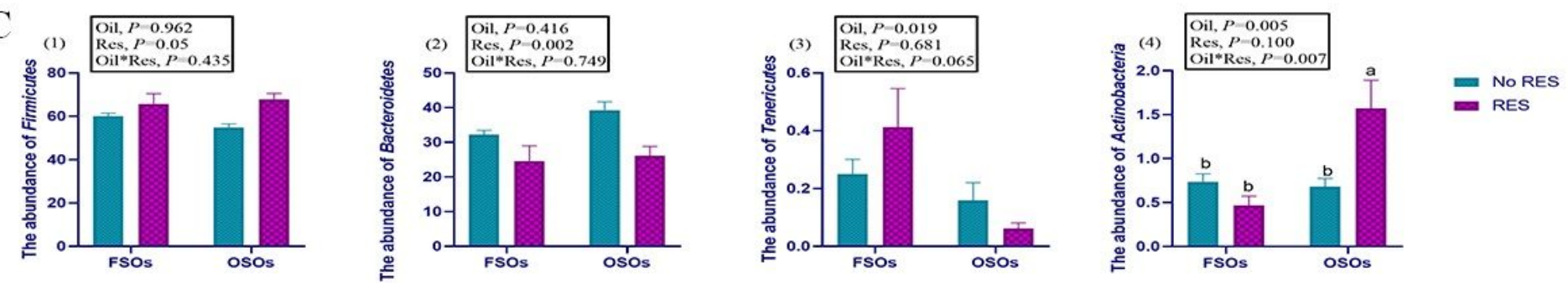

$\mathrm{D}$
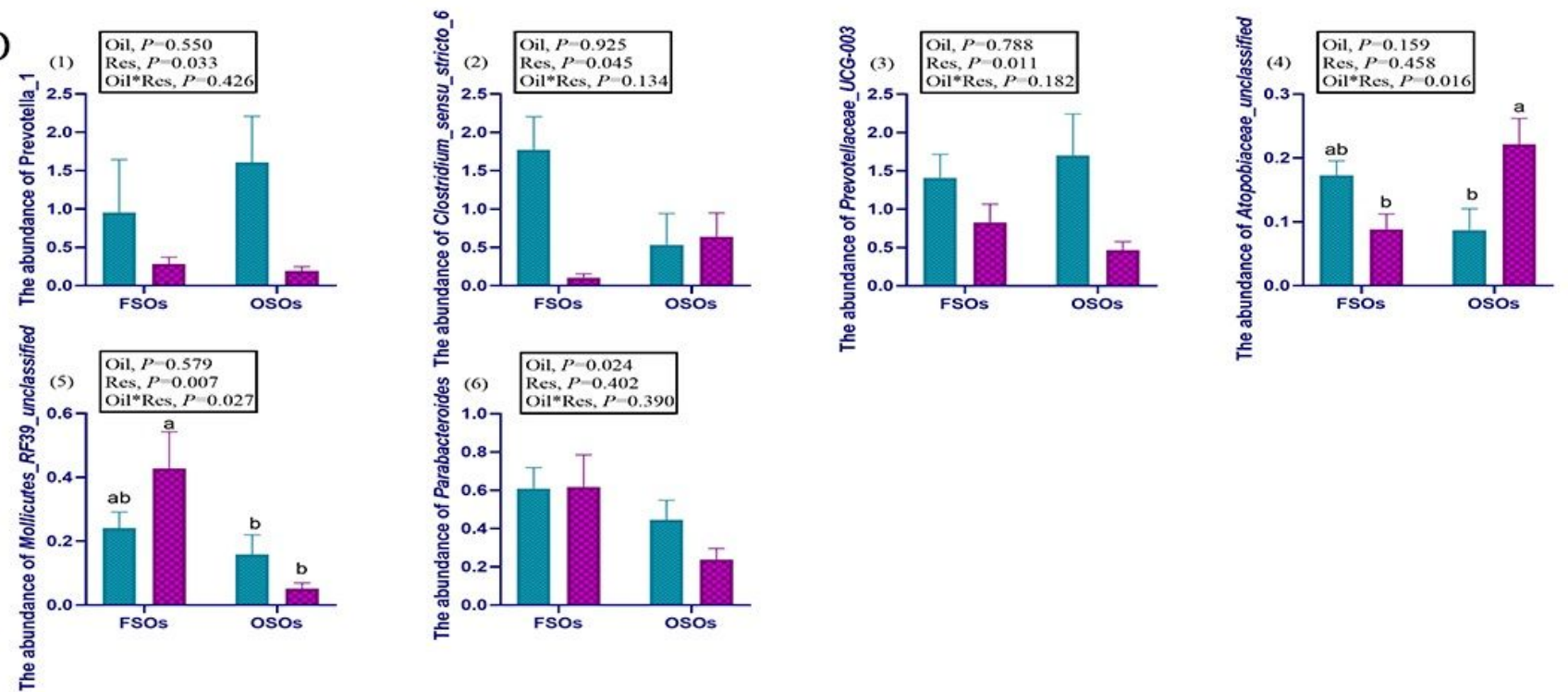

Figure 6 
Effects of OSOs and dietary RES on the fecal microbiota at the phylum and genus in the colon. $(\mathrm{A})$ at the phylum; (B)at the genus; (C-1) Firmicutes; (C-2) Bacteroidetes; (C-3) Tenericutes; (C-4) Actinobacteria; (C-5) Prevotella_1; (C-6) Clostridium; (C-7) Prevotellaceae_UCG-003; (C-8) Atopobiaceae_unclassified; (C-9) Mollicutes_RF39; (C-10) Parabacteroides. Note: FSOs, fresh soybean oils; FSOs+ RES, fresh soybean oils with resveratrol; OSOs, oxidized soybean oils; OSOs+ RES, oxidized soybean oils with resveratrol.

Different letters indicate that the change between each group is statistically significant $(P<0.05)$. Oil*RES, interaction between oxidized soybean oils and RES. The column and its bar represent the mean value and standard error ( $n=7$ piglets/group), respectively.
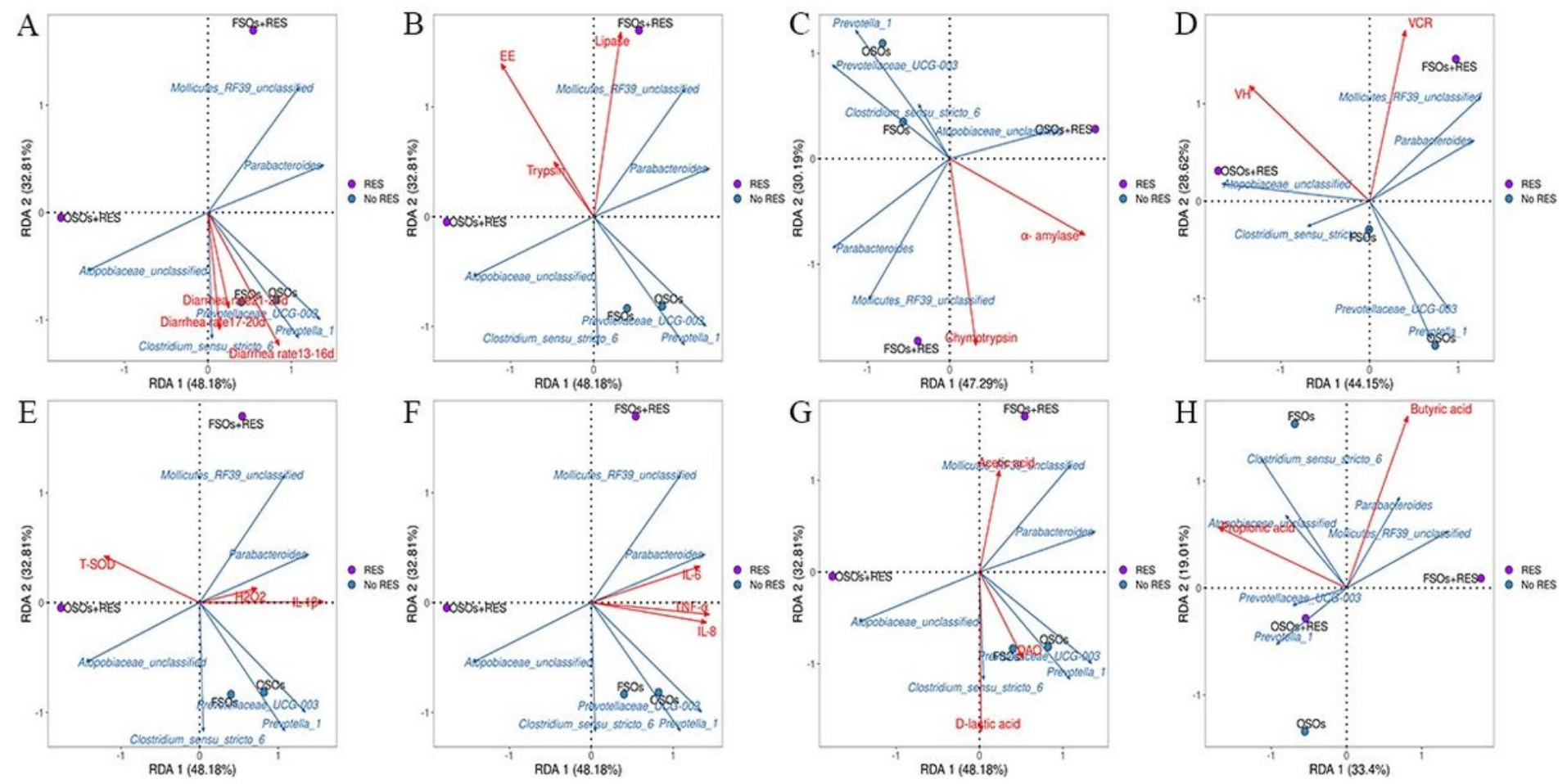

Figure 7

RDA analyses based on the identified differential genera of RES and OSOs revealed significantly altered apparent digestibility, proinflammatory factor and intestinal enzymatic activity-related indices. Note: FSOs, fresh soybean oils; FSOs+ RES, fresh soybean oils with resveratrol; OSOs, oxidized soybean oils; OSOs+ RES, oxidized soybean oils with resveratrol. For the relationship between groups and the identified altered phenotypes, if the plot projection of one sample occurred in the positive direction of the extending line of the identified altered phenotypes, then the treatment of this sample could promote this phenotypic change.

\section{Supplementary Files}

This is a list of supplementary files associated with this preprint. Click to download.

- Supplementarymaterial.docx 